Supplement of Geosci. Model Dev., 11, 4933-4964, 2018

https://doi.org/10.5194/gmd-11-4933-2018-supplement

(C) Author(s) 2018. This work is distributed under

the Creative Commons Attribution 4.0 License.

(c) (1)

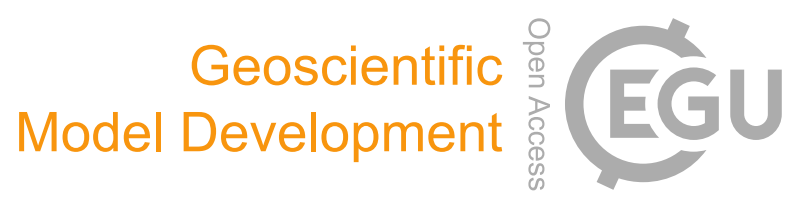

Supplement of

\title{
V2Karst V1.1: a parsimonious large-scale integrated vegetation-recharge model to simulate the impact of climate and land cover change in karst regions
}

Fanny Sarrazin et al.

Correspondence to: Fanny Sarrazin (fanny.sarrazin@bristol.ac.uk)

The copyright of individual parts of the supplement might differ from the CC BY 4.0 License. 


\section{S1. Challenges for modelling ET and representing land cover properties explicitly at large-scales}

Representing explicitly land cover properties for ET estimation requires the specification of vegetation properties, such as leaf area index, vegetation height, stomatal resistance, canopy interception storage capacity, and the availability of time series of climate variables such as air temperature, net radiation, humidity and wind speed. Modelling ET at large-scales faces a range of challenges: (1) a lack of ET observations to compare with model simulations, (2) a lack of observations of vegetation properties, and (3) uncertainty in large-scale forcing weather variables.

Firstly, on the ground, measurements of actual ET (e.g. FLUXNET network, Baldocchi et al., 2001) are limited in number and are only representative of plot scale ET. Their footprint can extend to a few hundred metres or possibly to a few kilometres (Baldocchi and Ryu, 2011), which is much smaller than the extent of typical largescale model simulation units that are mostly between $9 \mathrm{~km}$ ( $5^{\prime}$ grid) and $111 \mathrm{~km}$ ( $1^{\circ}$ grid) (Bierkens, 2015). Moreover, ground measurements of the partitioning of ET among its main components (transpiration, evaporation from interception and soil evaporation) are lacking as reported in Miralles et al. (2016) and in Fatichi and Pappas (2017) or are affected by large uncertainty (see e.g. Van Dijk et al., 2015 regarding evaporation from canopy interception), and the ET partitioning assessed using isotope techniques has large uncertainties and limited spatial coverage (Coenders-Gerrits et al., 2014; Sutanto et al., 2014). Additionally, global gridded ET products are available. Yet, these products do not provide direct observations of actual ET, but they are estimates of actual ET assessed using models that assimilate remote-sensed variables and either solve the energy balance or use potential ET (PET) equations as discussed e.g. in MacCabe (2016) and in Miralles et al. (2016). Jung et al. (2011) created a global gridded ET products based on model tree ensembles, which are trained using observations from the FLUXNET network.

A second issue is that observations of large-scale vegetation properties are limited. Large-scale gridded land cover databases provide spatially distributed information about the type of vegetation present around the world. We refer to Smith (2016) for a review of land cover databases. Large-scale gridded measurements of vegetation characteristics are obtained using remote-sensing techniques. Remote sensing techniques permit to retrieve vegetation leaf area index (LAI) (e.g. Fang et al., 2013) and other vegetation indices that can be only used as proxy for actual vegetation properties such as density or state of health, e.g. Vegetation Optical Depth (VOD), Normalized Difference Vegetation Index (NDVI) or Enhanced Vegetation Index (EVI) (see a review in Xue and $\mathrm{Su}, 2017)$. Moreover, such products suffer from a number of uncertainties, among which cloud contamination as reported e.g. in Fang et al. (2013) regarding LAI, and do not allow to assess critical vegetation properties such as rooting depth, stomatal resistance or canopy interception capacity. Ground measurements of vegetation properties are sparse and only few studies report collected values for specific variables or regions, these include Breuer et al. (2003) for a range of vegetation properties in temperate climates, Körner (1995) for stomatal resistance and Schenk and Jackson (2002) for rooting depth. Since ground measurements are limited, 
they do not allow to capture the variability in vegetation characteristics, as discussed in Wang-Erlandsson et al. (2016) regarding rooting depth measurements. In particular, stomatal resistance presents a high temporal variability because it is determined by weather conditions and therefore its measurements are particularly difficult to interpret (Breuer et al., 2003) and to use in modelling applications.

5 Thirdly, large-scale databases of historical weather data used to force model simulations are affected by large uncertainties because they have to rely on measurements with incomplete spatial coverage, in particular wind speed measurements (New et al., 2002). Moreover, the height from the ground at which these weather data are provided is uncertain. Measurements are assumed to be provided at standard heights, typically $10 \mathrm{~m}$ for wind speed and $2 \mathrm{~m}$ for temperature and humidity (see e.g. Rodell et al., 2004; Weedon et al., 2010), which may not 10 be representative of the specific location. 


\section{S2. Parameters used for ET estimation in large-scale models}

We reviewed the different approaches currently used to represent land cover properties explicitly in large-scale models, to assess their consistency with our three criteria for model development (Sect. 2.1) and to determine whether we could directly adopt some of these ET representations in the new version of the VarKarst model. In this section, we provide a detailed list of all parameters involved in the representation of ET in the largescale hydrological models we reviewed. These models are further described in Tables A1-A3.

\begin{tabular}{llllll}
\hline Parameter & Description & Module $^{\text {a }}$ & Category & Unit & Reference \\
\hline$Z_{r}$ & Rooting depth & Stress & Vegetation & {$[\mathrm{m}]$} & $\begin{array}{l}\text { (Vörösmarty et al., } \\
\text { 1989) } \\
\text { (Vörösmarty et al., }\end{array}$ \\
$A W C$ & Soil available water capacity & Stress & Soil & {$\left[\mathrm{m}^{3} \mathrm{~m}^{-3}\right]$} & $\begin{array}{l}\text { 1989) } \\
\text { (Vörösmarty et al., } \\
\alpha\end{array}$ \\
$\begin{array}{l}\text { Empirical coefficient of the drying curve } \\
\text { (set to 5) }\end{array}$ & Stress & Constant & {$[-]$} & 1998) \\
\hline
\end{tabular}

Table S1. Parameters used for ET estimation in the WBM model. The model includes a minimum of 3 parameters (reported in the table), and additional parameters depending on the PET formulation which is used (namely the Thornthwaite equation (Thornthwaite, 1948) in (Vörösmarty et al., 1996), the ShuttleworthWallace (Shuttleworth and Wallace, 1985) equation in (Federer et al., 2003), and a range of different PET equations in (Vörösmarty et al., 1998)).

${ }^{\text {a }}$ Stress: Stress model for actual ET calculation

\begin{tabular}{|c|c|c|c|c|c|}
\hline Parameter & Description & Module $^{\mathrm{a}}$ & Category & Unit & Reference \\
\hline$\beta_{28}$ & Aspect correction factor of PET & PET & Terrain & {$[-]$} & $\begin{array}{l}\text { (Kumar et al., } \\
\text { 2013; Samaniego } \\
\text { et al. 2010) }\end{array}$ \\
\hline$\beta_{1}$ & Effective maximum capacity storage & $\begin{array}{l}\text { Interception/ } \\
\text { Seasonality }\end{array}$ & Vegetation & {$[\mathrm{mm}]$} & $\begin{array}{l}\text { (Kumar et al., } \\
\text { 2013; Samaniego } \\
\text { et al., 2010) }\end{array}$ \\
\hline $\operatorname{Exp}_{\text {can }}$ & $\begin{array}{l}\text { Exponent to assess the wet canopy } \\
\text { fraction (set to } 2 / 3 \text { ) }\end{array}$ & Interception & Constant & {$[-]$} & $\begin{array}{l}\text { (Samaniego et al., } \\
\text { 2010) }\end{array}$ \\
\hline$\beta_{15}$ & Permanent wilting point & Stress & $\begin{array}{l}\text { Vegetation } \\
\text { and soil }\end{array}$ & {$[-]$} & $\begin{array}{l}\text { (Samaniego et al., } \\
\text { 2010) }\end{array}$ \\
\hline$\beta_{16}$ & $\begin{array}{l}\text { Soil moisture limit above which the actual } \\
\text { transpiration is equal to PET }\end{array}$ & Stress & $\begin{array}{l}\text { Vegetation } \\
\text { and soil }\end{array}$ & {$[-]$} & $\begin{array}{l}\text { (Samaniego et al., } \\
\text { 2010) }\end{array}$ \\
\hline$\beta_{17}^{1}$ & Fraction of roots in soil layer 1 & Stress & Vegetation & & $\begin{array}{l}\text { (Rakovec et al., } \\
\text { 2016; Samaniego } \\
\text { et al., 2010) }\end{array}$ \\
\hline$\beta_{17}^{2}$ & Fraction of roots in soil layer 2 & Stress & Vegetation & & $\begin{array}{l}\text { (Rakovec et al., } \\
\text { 2016; Samaniego } \\
\text { et al., 2010) }\end{array}$ \\
\hline$d_{1}$ & depth soil layer 1 (set to $0.05 \mathrm{~m}$ ) & Soil layers & Constant & {$[\mathrm{m}]$} & $\begin{array}{l}\text { (Rakovec et al., } \\
\text { 2016) }\end{array}$ \\
\hline$d_{2}$ & depth soil layer 2 (set to $0.25 \mathrm{~m}$ ) & Soil layers & Constant & {$[\mathrm{m}]$} & $\begin{array}{l}\text { (Rakovec et al., } \\
\text { 2016) }\end{array}$ \\
\hline$d_{3}$ & depth soil layer 3 (set to $1 \mathrm{~m}$ ) & Soil layers & Constant & {$[\mathrm{m}]$} & $\begin{array}{l}\text { (Rakovec et al., } \\
\text { 2016) }\end{array}$ \\
\hline
\end{tabular}

Table S2. Parameters used for ET estimation in the mHM model.

${ }^{a}$ PET: potential evapotranspiration equation; Stress: Stress model for actual ET calculation from PET. 


\begin{tabular}{|c|c|c|c|c|c|}
\hline Parameter & Description & Module $^{a}$ & Category & Unit & Reference \\
\hline$\alpha_{P T}$ & $\begin{array}{l}\text { Priestley-Taylor empirical coefficient } \\
\text { (1.26 in semiarid and arid areas and } 1.74 \\
\text { in humid areas) }\end{array}$ & PET & Climate & {$[-]$} & $\begin{array}{l}\text { (Döll et al., } \\
\text { 2003) }\end{array}$ \\
\hline$E_{\text {pot,max }}$ & $\begin{array}{l}\text { Maximum potential evapotranspiration } \\
\left(20 \mathrm{mmd}^{-1} \text { in semiarid and arid areas }\right. \\
\left.\text { and } 10 \mathrm{mmd}^{-1} \text { in humid areas }\right)\end{array}$ & Stress & Climate & {$\left[\mathrm{mm} \mathrm{d}^{-1}\right]$} & $\begin{array}{l}\text { (Müller Schmied } \\
\text { et al., 2014) }\end{array}$ \\
\hline$Z_{r}$ & Rooting depth & Stress & Vegetation & {$[\mathrm{m}]$} & $\begin{array}{l}\text { (Müller Schmied } \\
\text { et al., 2014) }\end{array}$ \\
\hline$A W C$ & Soil available water capacity & Stress & Soil & {$\left[\mathrm{m}^{3} \mathrm{~m}^{-3}\right]$} & $\begin{array}{l}\text { (Döll et al., } \\
\text { 2003) }\end{array}$ \\
\hline$V_{\text {can }}$ & $\begin{array}{l}\text { Interception storage capacity per unit of } \\
L A I \text { (set to } 0.3 \mathrm{~mm} \mathrm{LAI} \text { ) }\end{array}$ & Interception & Constant & $\begin{array}{l}{[\mathrm{mm}} \\
\mathrm{LAI}]\end{array}$ & $\begin{array}{l}\text { (Döll et al., } \\
\text { 2003) }\end{array}$ \\
\hline $\operatorname{Exp}_{\text {can }}$ & $\begin{array}{l}\text { Exponent to assess the wet canopy } \\
\text { fraction (set to } 2 / 3 \text { ) }\end{array}$ & Interception & Constant & {$[-]$} & $\begin{array}{l}\text { (Deardorff, } \\
\text { 1978; Döll et al., } \\
\text { 2003) }\end{array}$ \\
\hline$L A I_{\max }$ & Maximum leaf area index & Interception & Vegetation & {$\left[\mathrm{m}^{2} \mathrm{~m}^{-2}\right]$} & $\begin{array}{l}\text { (Müller Schmied } \\
\text { et al., 2014) }\end{array}$ \\
\hline$f_{d, l c}$ & $\begin{array}{l}\text { Fraction of deciduous plants in LAI } \\
\text { growth model }\end{array}$ & Seasonality & Vegetation & {$[-]$} & $\begin{array}{l}\text { (Müller Schmied } \\
\text { et al., 2014) }\end{array}$ \\
\hline$c_{e, l c}$ & $\begin{array}{l}\text { Reduction factor for evergreen plants in } \\
\text { LAI growth model }\end{array}$ & Seasonality & Vegetation & {$[-]$} & $\begin{array}{l}\text { (Müller Schmied } \\
\text { et al., 2014) }\end{array}$ \\
\hline$t_{\min }$ & $\begin{array}{l}\text { Initial days to start/end with growing } \\
\text { season in LAI growth model }\end{array}$ & Seasonality & Vegetation & [d] & $\begin{array}{l}\text { (Müller Schmied } \\
\text { et al., 2014) }\end{array}$ \\
\hline$L A I_{\min }$ & $\begin{array}{l}\text { Minimum leaf area index for deciduous } \\
\text { plants in LAI growth model (set to } 0.1 \\
\mathrm{~m}^{2} \cdot \mathrm{m}^{-2} \text { ) }\end{array}$ & Seasonality & Constant & {$\left[\mathrm{m}^{2} \mathrm{~m}^{-2}\right]$} & $\begin{array}{l}\text { (Müller Schmied } \\
\text { et al., 2014) }\end{array}$ \\
\hline$T_{\min }$ & $\begin{array}{l}\text { Daily temperature threshold to initiate } \\
\text { the growing season in LAI growth } \\
\text { model (set to } 8^{\circ} \mathrm{C} \text { ) }\end{array}$ & Seasonality & Constant & {$\left[{ }^{\circ} \mathrm{C}\right]$} & $\begin{array}{l}\text { (Müller Schmied } \\
\text { et al., 2014) }\end{array}$ \\
\hline$P_{\text {min }, \text { cum }}$ & $\begin{array}{l}\text { Cumulative precipitation threshold to } \\
\text { initiate the growing season in LAI } \\
\text { growth model (set to } 40 \mathrm{~mm} \text { ) }\end{array}$ & Seasonality & Constant & {$[\mathrm{mm}]$} & $\begin{array}{l}\text { (Müller Schmied } \\
\text { et al., 2014) }\end{array}$ \\
\hline$P_{\text {min, daily }}$ & $\begin{array}{l}\text { Minimum daily precipitation to keep } \\
\text { growing season growing in semi-arid } \\
\text { and arid regions in LAI growth model } \\
\text { (set to } 0.5 \mathrm{~mm} \text { ) }\end{array}$ & Seasonality & Constant & {$\left[\mathrm{mm} \mathrm{d}^{-1}\right]$} & $\begin{array}{l}\text { (Müller Schmied } \\
\text { et al., 2014) }\end{array}$ \\
\hline$t_{\text {growth }}$ & $\begin{array}{l}\text { Number of days for } L A I \text { to increase } \\
\text { from its minimum to its maximum value } \\
\text { or to decrease from its maximum to its } \\
\text { minimum value in LAI growth model } \\
\text { (set to } 30 \mathrm{~d} \text { ) }\end{array}$ & Seasonality & Constant & [d] & $\begin{array}{l}\text { (Müller Schmied } \\
\text { et al., 2014) }\end{array}$ \\
\hline
\end{tabular}

Table S3. Parameters used for ET estimation in the WaterGap V2.2 model.

${ }^{\text {a }}$ PET: potential evapotranspiration equation; Stress: Stress model for actual ET calculation from PET. 


\begin{tabular}{|c|c|c|c|c|c|}
\hline Parameter & Description & Module $^{\mathrm{a}}$ & Category & Unit & Reference \\
\hline$g_{\min }$ & Minimum canopy conductance & PET & Vegetation & {$\left[\mathrm{mm} \mathrm{s}^{-1}\right]$} & $\begin{array}{l}\text { (Gerten et al., } \\
\text { 2004; Sitch et al., } \\
\text { 2003) }\end{array}$ \\
\hline$g_{m}$ & $\begin{array}{l}\text { Scaling conductance in the } \\
\text { evaporative demand function (set } \\
\text { to } 3.26 \mathrm{~mm} \cdot \mathrm{s}^{-1} \text { ) }\end{array}$ & PET & Constant & {$\left[\mathrm{mm} \mathrm{s}^{-1}\right]$} & $\begin{array}{l}\text { (Gerten et al., } \\
\text { 2004) }\end{array}$ \\
\hline$\alpha_{m}$ & $\begin{array}{l}\text { Priestley-Taylor empirical } \\
\text { coefficient (set to 1.391) }\end{array}$ & PET & Constant & {$[-]$} & $\begin{array}{l}\text { (Gerten et al., } \\
\text { 2004) }\end{array}$ \\
\hline$\alpha_{P T}$ & $\begin{array}{l}\text { Priestley-Taylor empirical } \\
\text { coefficient (set to 1.32) }\end{array}$ & PET & Constant & {$[-]$} & $\begin{array}{l}\text { (Gerten et al., } \\
\text { 2004) }\end{array}$ \\
\hline$i$ & $\begin{array}{l}\text { Empirical coefficient for } \\
\text { calculation of interception (same } \\
\text { formulation as (Kergoat, 1998)) }\end{array}$ & Interception & Vegetation & {$[-]$} & $\begin{array}{l}\text { (Gerten et al., } \\
\text { 2004) }\end{array}$ \\
\hline$L A I$ & $\begin{array}{l}\text { Leaf area index (determined as a } \\
\text { function of daily phenomenology) }\end{array}$ & Interception & Vegetation & {$\left[\mathrm{m}^{2} \mathrm{~m}^{-2}\right]$} & $\begin{array}{l}\text { (Gerten et al., } \\
\text { 2004) }\end{array}$ \\
\hline$E_{\text {pot }, \max }$ & $\begin{array}{l}\text { Maximum potential } \\
\text { evapotranspiration }\left(5-7 \mathrm{~mm} . \mathrm{d}^{-1}\right)\end{array}$ & Stress & Vegetation & {$\left[\mathrm{mm} \mathrm{d}^{-1}\right]$} & $\begin{array}{l}\text { (Gerten et al., } \\
\text { 2004) }\end{array}$ \\
\hline$A W C$ & Soil available water capacity & Stress & Soil & {$\left[\mathrm{m}^{3} \mathrm{~m}^{-3}\right]$} & $\begin{array}{l}\text { (Gerten et al., } \\
\text { 2004) }\end{array}$ \\
\hline$f_{\text {root }, 0}$ & $\begin{array}{l}\text { Weighting constant to determine } \\
\text { fraction of roots in evaporation } \\
\text { layer (set to } 1.3 \text { ) }\end{array}$ & Stress & Constant & {$[-]$} & $\begin{array}{l}\text { (Gerten et al., } \\
\text { 2004) }\end{array}$ \\
\hline$f_{\text {root }, 1}$ & fraction of roots in soil layer 1 & Stress & Vegetation & {$[-]$} & $\begin{array}{l}\text { (Gerten et al., } \\
\text { 2004; Sitch et al., } \\
\text { 2003) }\end{array}$ \\
\hline$d_{1}$ & depth soil layer 1 (set to $0.5 \mathrm{~m}$ ) & Soil layers & Constant & {$[\mathrm{m}]$} & $\begin{array}{l}\text { (Gerten et al., } \\
\text { 2004) }\end{array}$ \\
\hline$d_{2}$ & depth soil layer 2 (set to $1 \mathrm{~m}$ ) & Soil layers & Constant & {$[\mathrm{m}]$} & $\begin{array}{l}\text { (Gerten et al., } \\
\text { 2004) }\end{array}$ \\
\hline$d_{0}$ & $\begin{array}{l}\text { depth evaporation layer (set to } 0.2 \\
\text { m) }\end{array}$ & Soil layers & Constant & {$[\mathrm{m}]$} & $\begin{array}{l}\text { (Gerten et al., } \\
\text { 2004) }\end{array}$ \\
\hline$f_{c}$ & $\begin{array}{l}\text { Vegetation cover fraction } \\
\text { (determined as a function of daily } \\
\text { phenomenology) }\end{array}$ & $\begin{array}{l}\text { Sparse } \\
\text { vegetation }\end{array}$ & Vegetation & {$[-]$} & $\begin{array}{l}\text { (Gerten et al., } \\
\text { 2004) }\end{array}$ \\
\hline
\end{tabular}

Table S4. Parameters used for ET estimation in the LPJ model.

${ }^{a}$ PET: potential evapotranspiration equation; Stress: Stress model for actual ET calculation from PET. 


\begin{tabular}{|c|c|c|c|c|c|}
\hline Parameter & Description & Module $^{\mathrm{a}}$ & Category & Unit & Reference \\
\hline$r_{a, v e g}$ & Vegetation aerodynamic resistance & PET & Vegetation & {$\left[\mathrm{s} \mathrm{m}^{-1}\right]$} & (Kergoat, 1998) \\
\hline$r_{s t}$ & Minimum stomatal resistance & PET & Vegetation & {$\left[\mathrm{s} \mathrm{m}^{-1}\right]$} & (Kergoat, 1998) \\
\hline$r_{a, s o i}$ & $\begin{array}{l}\text { Soil aerodynamic resistance (set to } 100 \\
\mathrm{~s} . \mathrm{m}^{-1} \text { ) }\end{array}$ & PET & Constant & {$\left[\mathrm{s} \mathrm{m}^{-1}\right]$} & (Kergoat, 1998) \\
\hline$r_{s, s o i}$ & Soil surface resistance (set to $50 \mathrm{~s} \cdot \mathrm{m}^{-1}$ ) & PET & Constant & {$\left[\mathrm{s} \mathrm{m}^{-1}\right]$} & (Kergoat, 1998) \\
\hline LAI & Leaf area index & $\begin{array}{l}\text { PET and } \\
\text { interception }\end{array}$ & Vegetation & {$\left[\mathrm{m}^{2} \mathrm{~m}^{-2}\right]$} & (Kergoat, 1998) \\
\hline$\beta$ & $\begin{array}{l}\text { Empirical coefficient for calculation of } \\
\text { interception }\end{array}$ & Interception & Vegetation & {$[-]$} & (Kergoat, 1998) \\
\hline$S_{1}$ & $\begin{array}{l}\text { Constant in radiation term in stomatal } \\
\text { resistance parameterization (set to } 10 \mathrm{~W} \\
\text { PAR.m }{ }^{-2)}\end{array}$ & $\begin{array}{l}\text { PET } \\
\text { (surface } \\
\text { resistance) }\end{array}$ & Constant & $\begin{array}{l}\text { [W PAR } \\
\left.\mathrm{m}^{-2}\right]\end{array}$ & (Kergoat, 1998) \\
\hline$f_{s}$ & $\begin{array}{l}\text { Fraction of photosynthetically active } \\
\text { solar radiation (set to } 0.48 \text { ) }\end{array}$ & $\begin{array}{l}\text { PET } \\
\text { (surface } \\
\text { resistance) }\end{array}$ & Constant & {$[-]$} & (Kergoat, 1998) \\
\hline$D_{1}$ & $\begin{array}{l}\text { First coefficient of the vapour pressure } \\
\text { deficit term in stomatal resistance } \\
\text { parameterization (set to } 3000 \mathrm{~Pa} \text { ) }\end{array}$ & $\begin{array}{l}\text { PET } \\
\text { (surface } \\
\text { resistance) }\end{array}$ & Constant & {$[\mathrm{Pa}]$} & (Kergoat, 1998) \\
\hline$D_{2}$ & $\begin{array}{l}\text { Second coefficient of the vapour } \\
\text { pressure deficit term in stomatal } \\
\text { resistance parameterization (set to } 3500 \\
\text { Pa) }\end{array}$ & $\begin{array}{l}\text { PET } \\
\text { (surface } \\
\text { resistance) }\end{array}$ & Constant & {$[\mathrm{Pa}]$} & (Kergoat, 1998) \\
\hline$k$ & $\begin{array}{l}\text { Beer- Lambert extinction coefficient (set } \\
\text { to } 0.5 \text { ) }\end{array}$ & $\begin{array}{l}\text { PET } \\
\text { (surface } \\
\text { resistance) } \\
\text { and Sparse } \\
\text { vegetation }\end{array}$ & Constant & {$[-]$} & (Kergoat, 1998) \\
\hline$Z_{r}$ & Rooting depth & Stress & Vegetation & {$[\mathrm{m}]$} & (Kergoat, 1998) \\
\hline$A W C$ & $\begin{array}{l}\text { Soil available water capacity } \\
\text { Soil water constant for stomatal closure }\end{array}$ & Stress & Soil & {$\left[\mathrm{m}^{3} \mathrm{~m}^{-3}\right]$} & (Kergoat, 1998) \\
\hline$W_{1}$ & $\begin{array}{l}\text { as a fraction of soil water storage (set to } \\
0.4 \text { ) }\end{array}$ & Stress & Constant & {$[-]$} & (Kergoat, 1998) \\
\hline$W_{2}$ & $\begin{array}{l}\text { Soil water constant for soil evaporation } \\
\text { reduction (set to 0.6) }\end{array}$ & Stress & Constant & {$[-]$} & (Kergoat, 1998) \\
\hline
\end{tabular}

Table S5. Parameters used for ET estimation in the model proposed by (Kergoat, 1998). We did not review the light limitation sub-model of the model, which is used to calculate an equilibrium value of LAI.

${ }^{a}$ PET: potential evapotranspiration equation; Stress: Stress model for actual ET calculation from PET. 


\begin{tabular}{|c|c|c|c|c|c|}
\hline Parameter & Description & Module $^{\mathrm{a}}$ & Category & Unit & Reference \\
\hline$K_{c}$ & $\begin{array}{l}\text { Crop factor (monthly values estimated } \\
\text { as a function of land cover and } \\
\text { climatology) }\end{array}$ & $\begin{array}{l}\text { PET (and } \\
\text { seasonality) }\end{array}$ & Vegetation & {$[-]$} & $\begin{array}{l}\text { (Van Beek, } \\
\text { 2008) }\end{array}$ \\
\hline$K_{c, \min }$ & $\begin{array}{l}\text { Minimum crop factor for bare soil (set } \\
\text { to } 0.2 \text { ) }\end{array}$ & PET & Constant & {$[-]$} & $\begin{array}{l}\text { (Van Beek, 2008; } \\
\text { Sperna Weiland } \\
\text { et al., 2015) }\end{array}$ \\
\hline$L A I$ & $\begin{array}{l}\text { Leaf area index (monthly values } \\
\text { estimated as a function of land cover } \\
\text { and climatology) }\end{array}$ & $\begin{array}{l}\text { Interception } \\
\text { (and } \\
\text { seasonality) }\end{array}$ & Vegetation & {$\left[\mathrm{m}^{2} \mathrm{~m}^{-2}\right]$} & $\begin{array}{l}\text { (Van Beek, 2008; } \\
\text { Sutanudjaja et } \\
\text { al., 2011) }\end{array}$ \\
\hline$V_{\text {can }}$ & $\begin{array}{l}\text { Interception storage capacity (set to } \\
0.3 \mathrm{~mm} \mathrm{LAI} \text { ) }\end{array}$ & Interception & Constant & $\begin{array}{l}{[\mathrm{mm}} \\
\mathrm{LAI}]\end{array}$ & $\begin{array}{l}\text { (Sutanudjaja et } \\
\text { al., 2011) }\end{array}$ \\
\hline$f_{\text {root }, 1}$ & Root fraction in soil layer 1 & Stress & Vegetation & {$[-]$} & $\begin{array}{l}\text { (Van Beek, 2008; } \\
\text { Sperna Weiland } \\
\text { et al., 2015; } \\
\text { Sutanudjaja et } \\
\text { al., 2011) }\end{array}$ \\
\hline$\beta_{1}$ & $\begin{array}{l}\text { Coefficient of the soil water retention } \\
\text { curve in soil layer } 1\end{array}$ & Stress & Soil & {$[-]$} & $\begin{array}{l}\text { (Van Beek, 2008; } \\
\text { Sutanudjaja et } \\
\text { al., 2011) }\end{array}$ \\
\hline$\beta_{2}$ & $\begin{array}{l}\text { Coefficient of the soil water retention } \\
\text { curve in soil layer } 2\end{array}$ & Stress & Soil & {$[-]$} & $\begin{array}{l}\text { (Van Beek, 2008; } \\
\text { Sutanudjaja et } \\
\text { al., 2011) }\end{array}$ \\
\hline$W_{\text {sat }, 1}$ & $\begin{array}{l}\text { Saturated volumetric moisture content } \\
\text { in soil layer } 1\end{array}$ & Stress & Soil & {$\left[\mathrm{m}^{3} \mathrm{~m}^{-3}\right]$} & $\begin{array}{l}\text { (Van Beek and } \\
\text { Bierkens, 2008; } \\
\text { Sperna Weiland } \\
\text { et al., 2015) }\end{array}$ \\
\hline$W_{\text {sat }, 2}$ & $\begin{array}{l}\text { Saturated volumetric moisture content } \\
\text { in soil layer } 2\end{array}$ & Stress & Soil & {$\left[\mathrm{m}^{3} \mathrm{~m}^{-3}\right]$} & $\begin{array}{l}\text { (Van Beek and } \\
\text { Bierkens, 2008; } \\
\text { Sperna Weiland } \\
\text { et al., 2015) }\end{array}$ \\
\hline$k_{s a t, 1}$ & $\begin{array}{l}\text { Saturated hydraulic conductivity in } \\
\text { soil layer } 1\end{array}$ & $\begin{array}{l}\text { Stress (soil } \\
\text { evaporation) }\end{array}$ & Soil & {$\left[\mathrm{m} \mathrm{d}^{-1}\right]$} & $\begin{array}{l}\text { (Van Beek, 2008; } \\
\text { Sutanudjaja et } \\
\text { al., 2011) }\end{array}$ \\
\hline$\Psi_{\text {sat }, 1}$ & $\begin{array}{l}\text { Matric soil suction at saturation in soil } \\
\text { layer } 1\end{array}$ & $\begin{array}{l}\text { Stress } \\
\text { (transpiration) }\end{array}$ & Soil & {$[\mathrm{m}]$} & $\begin{array}{l}\text { (Sutanudjaja et } \\
\text { al., 2011) }\end{array}$ \\
\hline$\Psi_{s a t, 2}$ & $\begin{array}{l}\text { Matric soil suction at saturation in soil } \\
\text { layer } 2\end{array}$ & $\begin{array}{l}\text { Stress } \\
\text { (transpiration) }\end{array}$ & Soil & {$[\mathrm{m}]$} & $\begin{array}{l}\text { (Sutanudjaja et } \\
\text { al., 2011) }\end{array}$ \\
\hline$\Psi_{50 \%}$ & $\begin{array}{l}\text { Matric soil suction at which } \\
\text { transpiration is halved (set for } \\
\text { instance equal to } 3.33 \mathrm{~m} \text { ) }\end{array}$ & $\begin{array}{l}\text { Stress } \\
\text { (transpiration) }\end{array}$ & Constant & {$[\mathrm{m}]$} & $\begin{array}{l}\text { (Sutanudjaja et } \\
\text { al., 2011) }\end{array}$ \\
\hline$d_{1}$ & Depth of soil layer 1 (set to $0.3 \mathrm{~m}$ ) & Stress & Constant & {$[\mathrm{m}]$} & $\begin{array}{l}\text { (Van Beek and } \\
\text { Bierkens, 2008) }\end{array}$ \\
\hline$d_{2}$ & Depth of soil layer 2 (set to $1.2 \mathrm{~m}$ ) & Stress & Constant & {$[\mathrm{m}]$} & $\begin{array}{l}\text { (Van Beek and } \\
\text { Bierkens, 2008) }\end{array}$ \\
\hline
\end{tabular}

Table S6. Parameters used for ET estimation in the PCR-GLOBWB model.

${ }^{\text {a }}$ PET: potential evapotranspiration equation; Stress: Stress model for actual ET calculation from PET. 


\begin{tabular}{|c|c|c|c|c|c|}
\hline Parameter & Description & Module $^{a}$ & Category & Unit & Reference \\
\hline$h_{\text {veg,over }}$ & Overstory vegetation height & PET & $\begin{array}{l}\text { Overstory } \\
\text { vegetation }\end{array}$ & {$[\mathrm{m}]$} & $\begin{array}{l}\text { (Gosling and } \\
\text { Arnell, 2011; } \\
\text { Smith, 2016) }\end{array}$ \\
\hline$r_{\text {st,over }}$ & $\begin{array}{l}\text { Overstory vegetation stomatal } \\
\text { resistance }\end{array}$ & PET & $\begin{array}{l}\text { Overstory } \\
\text { vegetation }\end{array}$ & {$\left[\mathrm{s} \mathrm{m}^{-1}\right]$} & $\begin{array}{l}\text { (Gosling and } \\
\text { Arnell, 2011; } \\
\text { Smith, 2016) }\end{array}$ \\
\hline$L A I_{\text {over }}$ & Overstory leaf area index & PET & $\begin{array}{l}\text { Overstory } \\
\text { vegetation }\end{array}$ & {$\left[\mathrm{m}^{2} \mathrm{~m}^{-2}\right]$} & $\begin{array}{l}\text { (Gosling and } \\
\text { Arnell, 2011; } \\
\text { Smith, 2016) }\end{array}$ \\
\hline$h_{\text {veg,over }}$ & $\begin{array}{l}\text { Understory vegetation height (set to } \\
\text { value for grass) }\end{array}$ & PET & $\begin{array}{l}\text { Understory } \\
\text { vegetation }\end{array}$ & {$[\mathrm{m}]$} & $\begin{array}{l}\text { (Gosling and } \\
\text { Arnell, 2011; } \\
\text { Smith, 2016) }\end{array}$ \\
\hline$r_{\text {st }, \text { under }}$ & $\begin{array}{l}\text { Understory vegetation stomatal } \\
\text { resistance (set to value for grass) }\end{array}$ & PET & $\begin{array}{l}\text { Understory } \\
\text { vegetation }\end{array}$ & {$\left[\mathrm{s} \mathrm{m}^{-1}\right]$} & $\begin{array}{l}\text { (Gosling and } \\
\text { Arnell, 2011; } \\
\text { Smith, 2016) }\end{array}$ \\
\hline$L A I_{\text {under }}$ & $\begin{array}{l}\text { Understory leaf area index (set to value } \\
\text { for grass) }\end{array}$ & PET & $\begin{array}{l}\text { Understory } \\
\text { vegetation }\end{array}$ & {$\left[\mathrm{m}^{2} \mathrm{~m}^{-2}\right]$} & $\begin{array}{l}\text { (Gosling and } \\
\text { Arnell, 2011; } \\
\text { Smith, 2016) }\end{array}$ \\
\hline$K$ & $\begin{array}{l}\text { Radiation coefficient to calculate } \\
\text { canopy surface resistance (set to } 0.7 \text { ) }\end{array}$ & PET & Constant & {$[-]$} & (Smith, 2016) \\
\hline$r_{s, s o i}$ & $\begin{array}{l}\text { (Soil) resistance to calculate canopy } \\
\text { surface resistance (set to } 100 \mathrm{~s} \cdot \mathrm{m}^{-1} \text { ) }\end{array}$ & PET & Constant & {$\left[\mathrm{s} \mathrm{m}^{-1}\right]$} & (Smith, 2016) \\
\hline$Z_{r, \text { over }}$ & Overstory rooting depth & Stress & $\begin{array}{l}\text { Overstory } \\
\text { vegetation }\end{array}$ & {$[\mathrm{m}]$} & $\begin{array}{l}\text { (Gosling and } \\
\text { Arnell, 2011; } \\
\text { Smith, 2016) }\end{array}$ \\
\hline$Z_{r, \text { under }}$ & $\begin{array}{l}\text { Understory rooting depth (set to value } \\
\text { for grass) }\end{array}$ & Stress & $\begin{array}{l}\text { Understory } \\
\text { vegetation }\end{array}$ & {$[\mathrm{m}]$} & $\begin{array}{l}\text { (Gosling and } \\
\text { Arnell, 2011; } \\
\text { Smith, 2016) } \\
\text { (Gosling and }\end{array}$ \\
\hline$F C$ & Soil field capacity & Stress & Soil & {$\left[\mathrm{m}^{3} \mathrm{~m}^{-3}\right]$} & $\begin{array}{l}\text { Arnell, 2011; } \\
\text { Smith, 2016) } \\
\text { (Gosling and }\end{array}$ \\
\hline$S_{\max }$ & Soil saturation capacity & Stress & Soil & {$\left[\mathrm{m}^{3} \mathrm{~m}^{-3}\right]$} & $\begin{array}{l}\text { Arnell, 2011; } \\
\text { Smith, 2016) }\end{array}$ \\
\hline$\gamma_{\text {over }}$ & Overstory interception capacity & Interception & $\begin{array}{l}\text { Overstory } \\
\text { vegetation }\end{array}$ & {$[\mathrm{mm}]$} & $\begin{array}{l}\text { (Gosling and } \\
\text { Arnell, 2011; } \\
\text { Smith, 2016) }\end{array}$ \\
\hline$\gamma_{\text {under }}$ & $\begin{array}{l}\text { Understory interception capacity (set to } \\
\text { value for grass) }\end{array}$ & Interception & $\begin{array}{l}\text { Understory } \\
\text { vegetation }\end{array}$ & {$[\mathrm{mm}]$} & $\begin{array}{l}\text { (Gosling and } \\
\text { Arnell, 2011; } \\
\text { Smith, 2016) }\end{array}$ \\
\hline$\delta$ & $\begin{array}{l}\text { Empirical parameter of interception } \\
\text { model (set to } 0.75 \text { ) }\end{array}$ & Interception & Constant & {$[-]$} & $\begin{array}{l}\text { (Arnell, 1999; } \\
\text { Smith, 2016) }\end{array}$ \\
\hline Percov & Percent overstory cover & $\begin{array}{l}\text { Sparse } \\
\text { vegetation }\end{array}$ & $\begin{array}{l}\text { Overstory } \\
\text { vegetation }\end{array}$ & {$[\%]$} & $\begin{array}{l}\text { (Gosling and } \\
\text { Arnell, 2011; } \\
\text { Smith, 2016) }\end{array}$ \\
\hline
\end{tabular}

Table S7. Parameters used for ET estimation in the Mac-PDM model.

a PET: potential evapotranspiration equation; Stress: Stress model for actual ET calculation from PET. 


\begin{tabular}{|c|c|c|c|c|c|}
\hline Parameter & Description & Module $^{a}$ & Category & Unit & Reference \\
\hline$z_{0}$ & Surface roughness length & PET & Vegetation & {$[\mathrm{m}]$} & $\begin{array}{l}\text { (Noilhan and } \\
\text { Planton, 1989) }\end{array}$ \\
\hline$r_{s t}$ & Minimum stomatal resistance & PET & Vegetation & {$\left[\mathrm{s} \mathrm{m}^{-1}\right]$} & $\begin{array}{l}\text { (Noilhan and } \\
\text { Planton, 1989) }\end{array}$ \\
\hline$L A I$ & $\begin{array}{l}\text { Leaf area index (average monthly } \\
\text { values) }\end{array}$ & $\begin{array}{l}\text { PET and } \\
\text { interception }\end{array}$ & Vegetation & {$\left[\mathrm{m}^{2} \mathrm{~m}^{-2}\right]$} & $\begin{array}{l}\text { (Noilhan and } \\
\text { Planton, 1989) }\end{array}$ \\
\hline$V_{\text {can }}$ & $\begin{array}{l}\text { Interception storage capacity per unit of } \\
L A I \text { (set to } 0.2 \mathrm{~mm} \mathrm{LAI} \text { ) }\end{array}$ & Interception & Constant & $\begin{array}{l}{[\mathrm{mm}} \\
\mathrm{LAI}]\end{array}$ & $\begin{array}{l}\text { (Noilhan and } \\
\text { Planton, 1989) }\end{array}$ \\
\hline $\operatorname{Exp}_{c a n}$ & $\begin{array}{l}\text { Exponent to assess the wet canopy } \\
\text { fraction (set to } 2 / 3 \text { ) }\end{array}$ & Interception & Constant & {$[-]$} & $\begin{array}{l}\text { (Deardorff, 1978; } \\
\text { Noilhan and } \\
\text { Planton, 1989) }\end{array}$ \\
\hline$R_{G L}$ & $\begin{array}{l}\text { Limit value of incoming solar radiation } \\
\text { (set to } 30 \mathrm{~W} \mathrm{~m}^{-2} \text { for forest and } 100 \mathrm{~W} \\
\mathrm{~m}^{-2} \text { for crop) }\end{array}$ & $\begin{array}{l}\text { PET } \\
\text { (surface } \\
\text { resistance) }\end{array}$ & Vegetation & {$\left[\mathrm{W} \mathrm{m} \mathrm{m}^{-2}\right]$} & $\begin{array}{l}\text { (Noilhan and } \\
\text { Planton, 1989) }\end{array}$ \\
\hline$r_{s t, \max }$ & $\begin{array}{l}\text { Maximum surface resistance (set to } \\
5000 \text { s. } \mathrm{m}^{-1} \text { ) }\end{array}$ & $\begin{array}{l}\text { PET } \\
\text { (surface } \\
\text { resistance) }\end{array}$ & Constant & {$\left[\mathrm{s} \mathrm{m}^{-1}\right]$} & $\begin{array}{l}\text { (Noilhan and } \\
\text { Planton, 1989) }\end{array}$ \\
\hline$f_{s}$ & $\begin{array}{l}\text { Fraction of photosynthetically active } \\
\text { solar radiation (set to } 0.55 \text { ) }\end{array}$ & $\begin{array}{l}\text { PET } \\
\text { (surface } \\
\text { resistance) }\end{array}$ & Constant & {$[-]$} & $\begin{array}{l}\text { (Noilhan and } \\
\text { Planton, 1989) }\end{array}$ \\
\hline$g$ & $\begin{array}{l}\text { Coefficient of the vapour pressure term } \\
\text { (set to } 0.025 \mathrm{hPa}^{-1} \text { ) }\end{array}$ & $\begin{array}{l}\text { PET } \\
\text { (surface } \\
\text { resistance) }\end{array}$ & Constant & {$\left[\mathrm{hPa}^{-1}\right]$} & $\begin{array}{l}\text { (Noilhan and } \\
\text { Planton, 1989) }\end{array}$ \\
\hline$k_{T}$ & $\begin{array}{l}\text { Coefficient of the temperature term (set } \\
\text { to } 0.0016 \mathrm{~K}^{-2} \text { ) }\end{array}$ & $\begin{array}{l}\text { PET } \\
\text { (surface } \\
\text { resistance) }\end{array}$ & Constant & {$\left[\mathrm{K}^{-2}\right]$} & $\begin{array}{l}\text { (Noilhan and } \\
\text { Planton, 1989) }\end{array}$ \\
\hline$W P$ & Wilting point volumetric water content & Stress & Soil & {$\left[\mathrm{m}^{3} \mathrm{~m}^{-3}\right]$} & $\begin{array}{l}\text { (Noilhan and } \\
\text { Planton, 1989) }\end{array}$ \\
\hline$W_{\text {sat }}$ & Saturated volumetric moisture content & Stress & Soil & {$\left[\mathrm{m}^{3} \mathrm{~m}^{-3}\right]$} & $\begin{array}{l}\text { (Noilhan and } \\
\text { Planton, 1989) }\end{array}$ \\
\hline$W_{\text {crit }}$ & Critical soil moisture (set to 0.75 ) & Stress & Constant & {$[-]$} & $\begin{array}{l}\text { (Noilhan and } \\
\text { Planton, 1989) }\end{array}$ \\
\hline$d_{1}$ & $\begin{array}{l}\text { Depth of the evaporation soil layer (set } \\
\text { to } 0.01 \mathrm{~m} \text { )) }\end{array}$ & Stress & Constant & {$[\mathrm{m}]$} & $\begin{array}{l}\text { (Noilhan and } \\
\text { Planton, 1989) }\end{array}$ \\
\hline$d_{2}$ & Rooting depth & Stress & Vegetation & {$[\mathrm{m}]$} & $\begin{array}{l}\text { (Noilhan and } \\
\text { Planton, 1989) }\end{array}$ \\
\hline$d_{3}$ & Total soil depth & Stress & $\begin{array}{l}\text { Vegetation } \\
\text { and soil }\end{array}$ & {$[\mathrm{m}]$} & $\begin{array}{l}\text { (Boone et al., } \\
\text { 1999) }\end{array}$ \\
\hline$f_{c}$ & Vegetation cover fraction & $\begin{array}{l}\text { Sparse } \\
\text { vegetation }\end{array}$ & Vegetation & {$[-]$} & $\begin{array}{l}\text { (Noilhan and } \\
\text { Planton, 1989) }\end{array}$ \\
\hline
\end{tabular}

Table S8. Parameters used for ET estimation in the ISBA model.

a PET: potential evapotranspiration equation; Stress: Stress model for actual ET calculation from PET. 


\begin{tabular}{|c|c|c|c|c|c|}
\hline Parameter & Description & Module $^{\mathrm{a}}$ & Category & Unit & Reference \\
\hline$\alpha_{P T}$ & Priestley-Taylor empirical coefficient & PET & Vegetation & {$[-]$} & $\begin{array}{l}\text { (Miralles et al., } \\
\text { 2011) }\end{array}$ \\
\hline$f_{G}$ & $\begin{array}{l}\text { Ground heat as a fraction of net } \\
\text { radiation }\end{array}$ & PET & Vegetation & {$[-]$} & $\begin{array}{l}\text { (Miralles et al., } \\
\text { 2011) }\end{array}$ \\
\hline$\beta$ & $\begin{array}{l}\text { Correction factor for transpiration to } \\
\text { account for hours with wet canopy (set } \\
\text { to } 0.07 \text { ) }\end{array}$ & $\begin{array}{l}\text { PET (tall } \\
\text { vegetation) }\end{array}$ & Constant & {$[-]$} & $\begin{array}{l}\text { (Miralles et al., } \\
\text { 2011) }\end{array}$ \\
\hline$V O D$ & $\begin{array}{l}\text { Vegetation optical depth (remotely } \\
\text { sensed) }\end{array}$ & $\begin{array}{l}\text { Stress and } \\
\text { seasonality }\end{array}$ & Vegetation & {$[-]$} & $\begin{array}{l}\text { (Martens et al., } \\
\text { 2017; Miralles et } \\
\text { al., 2011) }\end{array}$ \\
\hline$V O D_{\max }$ & Maximum vegetation optical depth & Stress & Vegetation & {$[-]$} & $\begin{array}{l}\text { (Martens et al., } \\
\text { 2017) }\end{array}$ \\
\hline$Z_{r}$ & Rooting depth & Stress & Vegetation & [m] & $\begin{array}{l}\text { (Miralles et al., } \\
\text { 2011) }\end{array}$ \\
\hline$W P$ & Wilting point & Stress & Soil & {$\left[\mathrm{m}^{3} \mathrm{~m}^{-3}\right]$} & $\begin{array}{l}\text { (Martens et al., } \\
\text { 2017) }\end{array}$ \\
\hline$F C$ & Field capacity & Stress & Soil & {$\left[\mathrm{m}^{3} \mathrm{~m}^{-3}\right]$} & $\begin{array}{l}\text { (Martens et al., } \\
\text { 2017) }\end{array}$ \\
\hline$S_{c}$ & $\begin{array}{l}\text { Canopy storage for tall vegetation (set } \\
\text { to } 1.2 \mathrm{~mm} \text { ) }\end{array}$ & $\begin{array}{l}\text { Interception } \\
\text { (tall } \\
\text { vegetation) }\end{array}$ & Constant & [mm] & $\begin{array}{l}\text { (Miralles et al., } \\
\text { 2010) }\end{array}$ \\
\hline$\overline{E_{c}}$ & $\begin{array}{l}\text { Mean evaporation rate for interception } \\
\text { for tall vegetation (set to } 0.3 \mathrm{~mm} \cdot \mathrm{h}^{-1} \text { ) }\end{array}$ & $\begin{array}{l}\text { Interception } \\
\text { (tall } \\
\text { vegetation) }\end{array}$ & Constant & {$\left[\mathrm{mm} \mathrm{h}^{-1}\right]$} & $\begin{array}{l}\text { (Miralles et al., } \\
\text { 2010) }\end{array}$ \\
\hline$\overline{R_{s}}$ & $\begin{array}{l}\text { Mean (synoptic) rainfall rate for tall } \\
\text { vegetation (set to } 1.5 \mathrm{~mm} \cdot \mathrm{h}^{-1} \text { ) }\end{array}$ & $\begin{array}{l}\text { Interception } \\
\text { (tall } \\
\text { vegetation) }\end{array}$ & Constant & {$\left[\mathrm{mm} \mathrm{h}^{-1}\right]$} & $\begin{array}{l}\text { (Miralles et al., } \\
\text { 2010) }\end{array}$ \\
\hline$\overline{R_{c}}$ & $\begin{array}{l}\text { Mean (convective) rainfall rate for tall } \\
\text { vegetation (set to } 5.6 \mathrm{~mm} \cdot \mathrm{h}^{-1} \text { ) }\end{array}$ & $\begin{array}{l}\text { Interception } \\
\text { (tall } \\
\text { vegetation) }\end{array}$ & Constant) & {$\left[\mathrm{mm} \mathrm{h}^{-1}\right]$} & $\begin{array}{l}\text { (Miralles et al., } \\
2010 \text { ) }\end{array}$ \\
\hline$p_{d}$ & $\begin{array}{l}\text { Fraction of rain to trunks for tall } \\
\text { vegetation (set to } 0.02 \text { ) }\end{array}$ & $\begin{array}{l}\text { Interception } \\
\text { (tall } \\
\text { vegetation) }\end{array}$ & Constant & {$[-]$} & $\begin{array}{l}\text { (Miralles et al., } \\
2010 \text { ) }\end{array}$ \\
\hline$e$ & $\begin{array}{l}\text { Fraction of trunk evaporation for tall } \\
\text { vegetation (set to } 0.02 \text { ) }\end{array}$ & $\begin{array}{l}\text { Interception } \\
\text { (tall } \\
\text { vegetation) }\end{array}$ & Constant & {$[-]$} & $\begin{array}{l}\text { (Miralles et al., } \\
\text { 2010) }\end{array}$ \\
\hline$S_{t}$ & $\begin{array}{l}\text { Trunk capacity for tall vegetation (set } \\
\text { to } 0.02 \mathrm{~mm} \text { ) }\end{array}$ & $\begin{array}{l}\text { Interception } \\
\text { (tall } \\
\text { vegetation) }\end{array}$ & Constant & {$[\mathrm{mm}]$} & $\begin{array}{l}\text { (Miralles et al., } \\
\text { 2010) }\end{array}$ \\
\hline$d_{1}$ & $\begin{array}{l}\text { Depth at the bottom of the first soil } \\
\text { layer (set to } 0.05 \mathrm{~m} \text { ) }\end{array}$ & Soil layers & Constant & {$[\mathrm{m}]$} & $\begin{array}{l}\text { (Miralles et al., } \\
\text { 2011) }\end{array}$ \\
\hline$d_{2}$ & $\begin{array}{l}\text { Depth at the bottom of the second soil } \\
\text { layer (set to } 1 \mathrm{~m} \text { ) }\end{array}$ & Soil layers & Constant & [m] & $\begin{array}{l}\text { (Miralles et al., } \\
\text { 2011) }\end{array}$ \\
\hline$d_{3}$ & $\begin{array}{l}\text { Depth at the bottom of the third soil } \\
\text { layer (set to } 2.5 \mathrm{~m} \text { ) }\end{array}$ & Soil layers & Constant & [m] & $\begin{array}{l}\text { (Miralles et al., } \\
\text { 2011) }\end{array}$ \\
\hline
\end{tabular}

Table S9. Parameters used for ET estimation in the GLEAM V3 model.

${ }^{a}$ PET: potential evapotranspiration equation; Stress model for actual ET calculation from PET. 


\begin{tabular}{|c|c|c|c|c|c|}
\hline Parameter & Description & Module $^{a}$ & Category & Unit & Reference \\
\hline$z_{0}$ & Surface roughness length & PET & Vegetation & {$[\mathrm{m}]$} & $\begin{array}{l}\text { (Liang et al., } \\
\text { 1994) }\end{array}$ \\
\hline$r_{s t}$ & Minimum stomatal resistance & PET & Vegetation & {$\left[\mathrm{s} \mathrm{m}^{-1}\right]$} & $\begin{array}{l}\text { (Bohn and } \\
\text { Vivoni, 2016; } \\
\text { Liang et al., } \\
\text { 1994) }\end{array}$ \\
\hline$r_{\text {arc }}$ & $\begin{array}{l}\text { Vegetation architectural resistance } \\
\text { (boundary layer resistance) }\end{array}$ & PET & Vegetation & {$\left[\mathrm{s} \mathrm{m}^{-1}\right]$} & $\begin{array}{l}\text { (Bohn and } \\
\text { Vivoni, 2016; } \\
\text { Liang et al., } \\
\text { 1994) }\end{array}$ \\
\hline$d_{0}$ & $\begin{array}{l}\text { Vegetation zero plane displacement } \\
\text { height }\end{array}$ & PET & Vegetation & {$[\mathrm{m}]$} & $\begin{array}{l}\text { (Liang et al., } \\
\text { 1994) }\end{array}$ \\
\hline$r_{s, \text { soi }}$ & Soil surface resistance (set to $0 \mathrm{~s} . \mathrm{m}^{-1}$ ) & PET & Constant & {$\left[\mathrm{s} \mathrm{m}^{-1}\right]$} & $\begin{array}{l}\text { (Bohn and } \\
\text { Vivoni, 2016) }\end{array}$ \\
\hline$r_{\text {arc }, \text { soi }}$ & $\begin{array}{l}\text { Soil architectural resistance (set to } 0 \\
\text { s. } \mathrm{m}^{-1} \text { ) }\end{array}$ & PET & Constant & {$\left[\mathrm{s} \mathrm{m}^{-1}\right]$} & $\begin{array}{l}\text { (Bohn and } \\
\text { Vivoni, 2016) } \\
\text { (Bohn and }\end{array}$ \\
\hline$L A I$ & $\begin{array}{l}\text { Leaf area index (average monthly } \\
\text { values) }\end{array}$ & $\begin{array}{l}\text { PET and } \\
\text { interception }\end{array}$ & Vegetation & {$\left[\mathrm{m}^{2} \mathrm{~m}^{-2}\right]$} & $\begin{array}{l}\text { Vivoni, 2016; } \\
\text { Liang et al., } \\
\text { 1994) }\end{array}$ \\
\hline$V_{\text {can }}$ & $\begin{array}{l}\text { Interception storage capacity per unit of } \\
L A I \text { (set to } 0.2 \mathrm{~mm} \mathrm{LAI} \text { ) }\end{array}$ & Interception & Constant & $\begin{array}{l}{[\mathrm{mm}} \\
\mathrm{LAI}]\end{array}$ & $\begin{array}{l}\text { (Liang et al., } \\
\text { 1994) }\end{array}$ \\
\hline $\operatorname{Exp}_{\text {can }}$ & $\begin{array}{l}\text { Exponent to assess the wet canopy } \\
\text { fraction (set to } 2 / 3 \text { ) }\end{array}$ & Interception & Constant & {$[-]$} & $\begin{array}{l}\text { (Deardorff, 1978; } \\
\text { Liang et al., } \\
\text { 1994) }\end{array}$ \\
\hline$R_{G L}$ & Limit value of incoming solar radiation & $\begin{array}{l}\text { PET } \\
\text { (surface } \\
\text { resistance) }\end{array}$ & Vegetation & {$\left[\mathrm{W} \mathrm{m} \mathrm{m}^{-2}\right]$} & $\begin{array}{l}\text { (Bohn and } \\
\text { Vivoni, 2016) }\end{array}$ \\
\hline$r_{s t, \max }$ & Maximum surface resistance & $\begin{array}{l}\text { PET } \\
\text { (surface } \\
\text { resistance) }\end{array}$ & Constant & {$\left[\mathrm{s} \mathrm{m}^{-1}\right]$} & $\begin{array}{l}\text { (Bohn and } \\
\text { Vivoni, 2016) }\end{array}$ \\
\hline$f_{s}$ & $\begin{array}{l}\text { Fraction of photosynthetically active } \\
\text { solar radiation }\end{array}$ & $\begin{array}{l}\text { PET } \\
\text { (surface } \\
\text { resistance) }\end{array}$ & Constant & {$[-]$} & $\begin{array}{l}\text { (Bohn and } \\
\text { Vivoni, 2016) }\end{array}$ \\
\hline$g$ & $\begin{array}{l}\text { Coefficient of the vapour pressure } \\
\text { deficit term }\end{array}$ & $\begin{array}{l}\text { PET } \\
\text { (surface } \\
\text { resistance) }\end{array}$ & Constant & {$\left[\mathrm{hPa}^{-1}\right]$} & $\begin{array}{l}\text { (Bohn and } \\
\text { Vivoni, 2016) }\end{array}$ \\
\hline$k_{T}$ & Coefficient of the temperature term & $\begin{array}{l}\text { PET } \\
\text { (surface } \\
\text { resistance) }\end{array}$ & Constant & {$\left[\mathrm{K}^{-2}\right]$} & $\begin{array}{l}\text { (Bohn and } \\
\text { Vivoni, 2016) }\end{array}$ \\
\hline$f_{\text {root }, 1}$ & Root fraction in first soil layer & Stress & Vegetation & {$[-]$} & $\begin{array}{l}\text { (Liang et al., } \\
\text { 1994) }\end{array}$ \\
\hline$W_{c r i t}$ & $\begin{array}{l}\text { Critical soil moisture in stomatal } \\
\text { resistance parameterization as a fraction } \\
\text { of soil saturation }\end{array}$ & Stress & Soil & {$\left[\mathrm{m}^{3} \mathrm{~m}^{-3}\right]$} & $\begin{array}{l}\text { (Bohn and } \\
\text { Vivoni, 2016; } \\
\text { Liang et al., } \\
\text { 1994) }\end{array}$ \\
\hline$W P$ & Wilting point & Stress & Soil & {$\left[\mathrm{m}^{3} \mathrm{~m}^{-3}\right]$} & $\begin{array}{l}\text { (Bohn and } \\
\text { Vivoni, 2016; } \\
\text { Liang et al., } \\
\text { 1994) }\end{array}$ \\
\hline$d_{1}$ & Depth of soil layer 1 (e.g. set to $0.3 \mathrm{~m}$ ) & Stress & Constant & {$[\mathrm{m}]$} & $\begin{array}{l}\text { (Liang et al., } \\
\text { 1994) }\end{array}$ \\
\hline$d_{2}$ & Depth of soil layer 2 (e.g. set to $0.7 \mathrm{~m}$ ) & Stress & Constant & {$[\mathrm{m}]$} & $\begin{array}{l}\text { (Liang et al., } \\
\text { 1994) }\end{array}$ \\
\hline$N D V I$ & $\begin{array}{l}\text { Normalized Difference Vegetation } \\
\text { Index (remotely sensed daily values) }\end{array}$ & $\begin{array}{l}\text { Sparse } \\
\text { vegetation } \\
\text { and } \\
\text { seasonality }\end{array}$ & Vegetation & {$[-]$} & $\begin{array}{l}\text { (Bohn and } \\
\text { Vivoni, 2016) }\end{array}$ \\
\hline$N D V I_{\min }$ & $\begin{array}{l}\text { Minimum Normalized Difference } \\
\text { Vegetation Index (set to } 0.1 \text { ) }\end{array}$ & $\begin{array}{l}\text { Sparse } \\
\text { vegetation }\end{array}$ & Constant & {$[-]$} & $\begin{array}{l}\text { (Bohn and } \\
\text { Vivoni, 2016) }\end{array}$ \\
\hline
\end{tabular}




\begin{tabular}{llllll}
\hline \multirow{2}{*}{$N D V I_{\max }$} & $\begin{array}{l}\text { Maximum Normalized Difference } \\
\text { Vegetation Index (set to 0.8) }\end{array}$ & $\begin{array}{l}\text { Sparse } \\
\text { vegetation }\end{array}$ & Constant & {$[-]$} & $\begin{array}{l}\text { (Bohn and } \\
\text { Vivoni, 2016) }\end{array}$ \\
\hline
\end{tabular}

Table S10. Parameters used for ET estimation in the VIC V4.2 model. Additional information on model parameters was found in the GLDAS project (https://ldas.gsfc.nasa.gov/gldas/GLDASmapveg.php).

a PET: potential evapotranspiration equation; Stress: Stress model for actual ET calculation from PET. 


\section{S3. Additional information on the determination of parameter ranges}

In this section, Tables S11 and S12 are extended versions of Tables 1 and 3 respectively that present the model parameters and the ranges used for application of V2Karst at FLUXNET sites. We added explanations and references for the determination of the parameter ranges. 


\begin{tabular}{|c|c|c|c|c|c|c|}
\hline Parameter & Description & unit & $\begin{array}{c}\text { Lower } \\
\text { limit }\end{array}$ & $\begin{array}{l}\text { Upper } \\
\text { limit }\end{array}$ & Category & Note and references for parameter range \\
\hline$h_{v e g}$ & Vegetation height & {$[\mathrm{m}]$} & 0.2 & $\begin{array}{c}\text { Site } \\
\text { specific }\end{array}$ & vegetation & $\begin{array}{l}\text { The upper bound is set for each site specifically so that it is lower than the } \\
\text { measurement heights reported in Table B1. }\end{array}$ \\
\hline$r_{s t}$ & Stomatal resistance & {$\left[\mathrm{s} \mathrm{m}^{-1}\right]$} & 20 & 600 & vegetation & $\begin{array}{l}\text { The range includes the 70th percentiles of the values for the different vegetation } \\
\text { types in temperate climate (Breuer et al., 2003). }\end{array}$ \\
\hline$L A I_{\min }$ & $\begin{array}{l}\text { Reduction in leaf area index } \\
\text { during the dormant season }\end{array}$ & {$[\%]$} & 5 & 100 & vegetation & Best guess estimate. \\
\hline$L A I_{\max }$ & $\begin{array}{l}\text { Annual maximum leaf area } \\
\text { index }\end{array}$ & {$\left[\mathrm{m}^{2} \mathrm{~m}^{-2}\right]$} & 0.5 & 8 & vegetation & $\begin{array}{l}\text { The range includes the 70th percentiles calculated for the different vegetation types } \\
\text { in temperate climate (Breuer et al., 2003). }\end{array}$ \\
\hline$V_{r}$ & $\begin{array}{l}\text { Maximum storage capacity of } \\
\text { the root zone }\end{array}$ & {$[\mathrm{mm}]$} & 20 & 500 & vegetation & $\begin{array}{l}\text { The range includes the 70th percentiles of the values of rooting depth (provided in } \\
\text { [m]) for the different vegetation types in temperate climate (Breuer et al., 2003) } \\
\text { multiplied by an average value of soil available water capacity of } 0.2 \mathrm{~m}^{3} \mathrm{~m}^{-3} \text { (Bonan, } \\
\text { 2015; Miralles et al., 2011; Salter and Williams, 1965). }\end{array}$ \\
\hline$V_{\text {can }}$ & $\begin{array}{l}\text { Canopy storage capacity per } \\
\text { unit of } L A I\end{array}$ & $\begin{array}{l}{[\mathrm{mm}} \\
\mathrm{LAI}]\end{array}$ & 0.1 & 0.5 & vegetation & $\begin{array}{l}\text { The range includes the value used in WaterGap (Döll et al., 2003) for daily } \\
\text { application (0.3 mm LAI); in VIC (Liang et al., 1994) and ISBA (Noilhan and } \\
\text { Planton, 1989) for subdaily applications as proposed in (Dickinson, 1984) }(0.2 \mathrm{~mm} \\
\text { LAI); in the Distributed Hydrology-Soil-Vegetation model (Wigmosta et al., 1994) } \\
\text { for subdaily applications (0.1 mm LAI); the maximum value used in Mac-PDM } \\
\text { [Gosling and Arnell, 2011] (0.5 mm LAI for open shrublands). }\end{array}$ \\
\hline$k$ & $\begin{array}{l}\text { Beer-Lambert's law extinction } \\
\text { coefficient }\end{array}$ & {$[-]$} & 0.4 & 0.7 & vegetation & $\begin{array}{l}\text { The range includes the value reported in (Van Dijk and Bruijnzeel, 2001; Granier et } \\
\text { al., 1999; Kergoat, 1998; Ruiz et al., 2010) (0.5); in (Shuttleworth and Wallace, } \\
\text { 1985) (0.7). }\end{array}$ \\
\hline$f_{\text {red }}$ & $\begin{array}{l}\text { Reduction factor for } \\
\text { transpiration below the root } \\
\text { zone }\end{array}$ & {$[-]$} & 0 & 0.15 & soil & $\begin{array}{l}\text { The range includes the value reported in (Penman, 1950; Wagener et al., 2003) } \\
(1 / 12) \text {. }\end{array}$ \\
\hline$z_{0}$ & Soil roughness length & {$[\mathrm{m}]$} & 0.0003 & 0.013 & soil & $\begin{array}{l}\text { The range includes the value used in MOSES (Essery et al., 2001) }(0.0003 \mathrm{~m}) \text {; in } \\
\text { Hydrus (Šimůnek et al., 2009) }(0.001 \mathrm{~m}) \text {; in NOAH (Yang et al., 2011) and the } \\
\text { Community Land model (Oleson et al., 2010) (0.01 m); in (Masson et al., 2003) } \\
(0.013 \mathrm{~m}) \text {. }\end{array}$ \\
\hline$r_{s, \text { soi }}$ & Soil surface resistance & {$\left[\mathrm{s} \mathrm{m}^{-1}\right]$} & 0 & 100 & soil & $\begin{array}{l}\text { The range includes the value used in VIC (Bohn and Vivoni, 2016) and SWAP } \\
\text { (Kroes et al., 2008) }\left(0 \mathrm{~m} \mathrm{~s}^{-1}\right) \text {; in (Kergoat, 1998) }\left(50 \mathrm{~m} \mathrm{~s}^{-1}\right) \text {; in MacPDM (Smith, } \\
\text { 2016) }\left(100 \mathrm{~m} \mathrm{~s}^{-1}\right) \text {; in }\left(\text { Van de Griend and Owe, 1994) }\left(10 \mathrm{~m} \mathrm{~s}^{-1}\right) \text {. }\right.\end{array}$ \\
\hline$V_{e}$ & $\begin{array}{l}\text { Maximum storage capacity of } \\
\text { the first soil layer }\end{array}$ & {$[\mathrm{mm}]$} & 5 & 45 & soil & $\begin{array}{l}\text { Range includes the average depth of } 0.1-0.15 \mathrm{~m} \text { recommended in (Allen et al., 1998) } \\
\text { multiplied by a large value of the soil water capacity of } 0.3 \mathrm{~m}^{3} \mathrm{~m}^{-3} \text { ((Bonan, 2015; } \\
\text { Salter and Williams, 1965)). }\end{array}$ \\
\hline$a$ & Spatial variability coefficient & {$[-]$} & 0 & 6 & $\begin{array}{l}\text { soil and } \\
\text { epikarst }\end{array}$ & (Hartmann et al., 2015) \\
\hline$V_{\text {soil }}$ & Mean soil storage capacity & {$[\mathrm{mm}]$} & 20 & 800 & & Best guess estimate. \\
\hline$V_{e p i}$ & Mean epikarst storage capacity & {$[\mathrm{mm}]$} & 200 & 700 & epikarst & (Hartmann et al., 2015) \\
\hline$K_{\text {epi }}$ & $\begin{array}{l}\text { Mean epikarst outflow } \\
\text { coefficient }\end{array}$ & [d] & 0 & 50 & epikarst & (Hartmann et al., 2015) \\
\hline
\end{tabular}


Table S11. Description of V2Karst parameters, unconstrained ranges used in the application at the four FLUXNET sites to capture the variability across soil, epikarst and vegetation types, category of the parameters (which indicated whether the parameters depend on soil, epikarst or vegetation properties) and references for the determination of parameter ranges. Parameters $a, V_{\text {soil }}, V_{\text {epi }}$ and $K_{\text {epi }}$ were already present in the previous version of the model (VarKarst). 


\begin{tabular}{|c|c|c|c|c|c|c|c|c|c|c|}
\hline \multirow[t]{2}{*}{ Parameter } & \multirow[t]{2}{*}{ Unit } & \multicolumn{2}{|c|}{$\begin{array}{l}\text { German site } \\
\text { (deciduous } \\
\text { forest) }\end{array}$} & \multicolumn{2}{|c|}{$\begin{array}{l}\text { Spanish site } \\
\text { (shrubland) }\end{array}$} & \multicolumn{2}{|c|}{$\begin{array}{l}\text { French } 1 \text { site } \\
\text { (evergreen } \\
\text { forest) }\end{array}$} & \multicolumn{2}{|c|}{$\begin{array}{l}\text { French } 2 \text { site } \\
\text { (evergreen } \\
\text { forest) }\end{array}$} & \multirow[t]{2}{*}{ Note and reference for parameter ranges } \\
\hline & & $\begin{array}{l}\text { Lower } \\
\text { limit }\end{array}$ & $\begin{array}{l}\text { Upper } \\
\text { limit }\end{array}$ & $\begin{array}{c}\text { Lower } \\
\text { limit }\end{array}$ & $\begin{array}{l}\text { Upper } \\
\text { limit }\end{array}$ & $\begin{array}{c}\text { Lower } \\
\text { limit }\end{array}$ & $\begin{array}{l}\text { Upper } \\
\text { limit }\end{array}$ & $\begin{array}{l}\text { Lower } \\
\text { limit }\end{array}$ & $\begin{array}{l}\text { Upper } \\
\text { limit }\end{array}$ & \\
\hline$h_{v e g}$ & {$[\mathrm{~m}]$} & 23.1 & 42.9 & 0.35 & 0.85 & 7.1 & 13.3 & 3.9 & 7.2 & $\begin{array}{l}\text { The range corresponds to the average value reported in Table B } 1 \text { for } \\
\text { the site } \pm 30 \% \text {. At the Spanish site, the upper bound is set higher due } \\
\text { to the presence of a few plants taller than average. }\end{array}$ \\
\hline$r_{s t}$ & {$\left[\mathrm{~s} \mathrm{~m}^{-1}\right]$} & 275 & 400 & 195 & 350 & 320 & 455 & 320 & 455 & $\begin{array}{l}40^{\text {th }} \text { and } 60^{\text {th }} \text { percentile values reported in (Breuer et al., 2003) for } \\
\text { the specific land cover at the site. }\end{array}$ \\
\hline$L A I_{\min }$ & {$[\%]$} & 5 & 20 & 34 & 63 & 80 & 100 & 80 & 100 & $\begin{array}{l}\text { At the Spanish site, the range corresponds to the value reported in } \\
\text { Table B } 1 \text { for the site } \pm 30 \% \text {, and it is a best guess estimates for the } \\
\text { other sites. }\end{array}$ \\
\hline$L A I_{\max }$ & {$\left[\mathrm{m}^{2} \mathrm{~m}^{-2}\right]$} & 3.5 & 6.5 & 1.9 & 3.5 & 1.5 & 2.9 & 2.0 & 3.8 & $\begin{array}{l}\text { The range corresponds to the value reported in Table B1 for the site } \\
\pm 30 \% \text {. }\end{array}$ \\
\hline$V_{r}$ & {$[\mathrm{~mm}]$} & 60 & 300 & 30 & 200 & 30 & 200 & 30 & 200 & $\begin{array}{l}\text { The range includes the average value of the soil available water } \\
\text { capacity for the German, Spanish and French } 2 \text { sites, and the value } \\
\text { of the available water capacity of the root zone for the French } 2 \text { site. } \\
\text { The upper bound is set to a high value to include uncertainty and to } \\
\text { account for the fact that at the German, Spanish and French } 1 \text { sites, } \\
\text { roots could extend below the soil because the soil is quite shallow. } \\
\text { Best guess estimates. }\end{array}$ \\
\hline
\end{tabular}

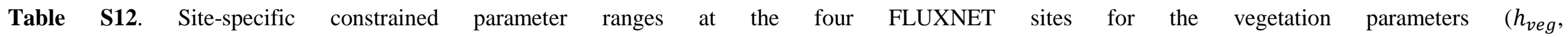
$\left.r_{s t}, L A I_{\min }, L A I_{\max }, V_{r}\right)$ and for the soil storage capacity $\left(V_{s o i}\right)$ and references for the determination of parameter ranges. 


\section{S4. Data processing and analysis at FLUXNET sites}

This section provides details on the processing of the data measured at the FLUXNET site to force and test the V2Karst model and to perform the virtual experiments (this section complements Sect. 3.2 and Sect. 4.3).

\section{S4.1 Processing of forcing data}

Measurements of precipitation, air temperature, net radiation, relative humidity and wind speed were gap-filled and then aggregated from $30 \mathrm{~min}$ to daily time scale. Missing precipitation data were filled with zero values for short gaps only (less or equal to 3 hours). For all other variables, we used the following procedure for gapfilling:

- $\quad$ short gaps (less or equal to 3 hours) were filled using linear interpolation;

- medium gaps (from 3.5 hours to 15 days) were filled using moving window averaging, i.e. the values corresponding to same time of the day for the previous and following days were averaged. For each gap we expanded progressively the width of the moving window until a minimum of four values to calculate the average were found. The maximum width of the moving window was 30 days.

- long gaps (from 15 to 80 days) were filled using long term averaging, i.e. for each month, we derived an average value for each time of the day by calculating the average over the entire time series.

We could then extract for each site a simulation period for which no gaps remained. We identified the 'poor' months for which the forcing data contained many gaps, and therefore for which the impact of the gap-filling on the simulation results is likely to be significant. 'Poor' months were defined as the months that had more than $20 \%$ of the days that contained gap-filled data. In addition, after each period of months that contained many gaps, we also discarded (added to the list of 'poor' months') a period of the same length because we assumed that the impact of the gap-filling is still significant over this subsequent period. During the 'poor' months we did not compare model simulations with latent heat flux and soil moisture observations when applying the soft rules for parameter estimation (Sect. 4.1).

\section{S4.2 Processing and analysis of the uncertainty in observed ET}

\section{Processing of latent heat flux measurements}

Observations of latent heat flux were aggregated from $30 \mathrm{~min}$ to monthly time scale and we discarded the months when more than $20 \%$ of 30 min data were missing. We also removed monthly aggregated latent heat flux measurements when the mismatch in the energy balance closure was higher than $50 \%$ similar to Miralles et al. (2011). We also derived two corrected estimates of actual ET, obtained by forcing the closure in the energy balance following Twine et al. (2000) and Foken et al. (2012):

1. a corrected value that assumes that latent heat flux $\left(L E\left[\mathrm{MJ} \mathrm{m}^{-2} \mathrm{month}^{-1}\right]\right)$ and sensible heat flux $\left(H\left[\mathrm{MJ} \mathrm{m}^{-2}\right.\right.$ month $\left.\left.^{-1}\right]\right)$ have similar errors (referred to as Bowen ratio estimate, $\left.E_{\text {act,cor }}\left[\mathrm{mm} \mathrm{month}^{-1}\right]\right)$ : 


$$
E_{\text {act }, \mathrm{cor}}=\frac{R_{n}-G}{\lambda\left(1+\frac{H}{L E}\right)},
$$

where $R_{n}\left[\mathrm{MJ} \mathrm{m}{ }^{-2}\right.$ month $\left.^{-1}\right]$ is the net radiation, $G\left[\mathrm{MJ} \mathrm{m}{ }^{-2}\right.$ month $\left.^{-1}\right]$ is the ground heat flux and $\lambda\left[\mathrm{MJ} \mathrm{kg}^{-1}\right]$ is the latent heat of vaporization of water;

2. a second corrected value that assumes errors in latent heat flux only (referred to as residual estimate, $E_{\text {act,cor } 2}\left[\mathrm{~mm}\right.$ month $\left.\left.^{-1}\right]\right)$ :

$$
E_{\text {act,cor } 2}=\frac{R_{n}-G-H}{\lambda} \text {. }
$$

Impact of neglecting the ground heat flux in Eq. (S1-S2)

We note that measurements of $G$ are not available for the French 1 site and contain many gaps for the French 2 site. Therefore, the tested the impact of neglecting the ground heat flux $G$ in Eq. (S1-S2), i.e. of setting $G=$ 0 . We assessed the closure in the energy balance and the two correct estimates of ET (Eq. (S1-S2)) in two cases, a first case in which we included the measurements of $G$ and a second case in which we neglected them. We conducted this analysis at the FLUXNET sites for which measurements of $G$ are available (German, Spanish and French 2 site). The monthly time series of the two corrected estimates of ET of Eq. (S1-S2) obtained are reported in Fig. S1.

We then assessed the bias Bias $_{1}[\%]$ and the monthly Pearson correlation coefficient $\rho_{1}[-]$ between the corrected estimate of Eq. (S1) processed with and without measurements of $G$ :

$$
\operatorname{Bias}_{1}=100 \frac{\sum_{t \in M_{E T}}\left(E_{a c t, c o r}^{n o G}(t)-E_{a c t, c o r}^{G}(t)\right)}{\sum_{t \in M_{E T}} E_{a c t, c o r}^{G}(t)}
$$

15 where $E_{\text {act,cor }}^{n o G}$ is the estimate of Eq. (S1) assessed neglecting $G, E_{\text {act,cor }}^{G}$ is the estimate of Eq. (S1) assessed using $G$ measurements and $M_{E T}$ is the set of months for which latent heat flux measurements are available and for which the simulations can be compared to the observations because the forcing data contain few gaps (blue areas in Fig. S1).

Table S13 shows the values of Bias ${ }_{1}$ and $\rho_{1}$ at the German, Spanish and French 2 sites. We see that $\rho_{1}$ is close to 1 , and therefore neglecting $G$ does not impact the dynamic of the ET estimate. Bias $_{1}$ is generally very small $(<2 \%)$ apart from the Spanish site where it is equal to $-8.3 \%$. This is due to the fact that a small number of observations can be used at the Spanish site and that Bias 1 is therefore largely impacted by some differences between $E_{a c t, c o r}^{n o G}$ and $E_{\text {act,cor }}^{G}$ at the end of year 2010 (Fig. S1).

Our analysis shows that it is reasonable to neglect the ground heat flux (i.e. to assume $G=0$ ) when assessing the corrected estimates of ET. This allows to use the French 1 site to test the model and to use a longer time series of ET measurements to test the model at the French 2 site. 


\begin{tabular}{lccc}
\hline Site & Number of monthly values used to assess Bias $_{1}$ and $\rho_{1}$ & Bias $_{1}[\%]$ & $\rho_{1}[-]$ \\
\hline German site & 61 & 1.5 & 1 \\
Spanish site & 12 & -8.3 & 0.99 \\
French 2 site & 22 & 0.2 & 1 \\
\hline
\end{tabular}

Table S13. Bias Bias 1 and monthly correlation coefficient $\rho_{1}$ between the monthly Bowen ratio estimate of Eq. (S1) assessed when neglecting or using the ground heat flux.
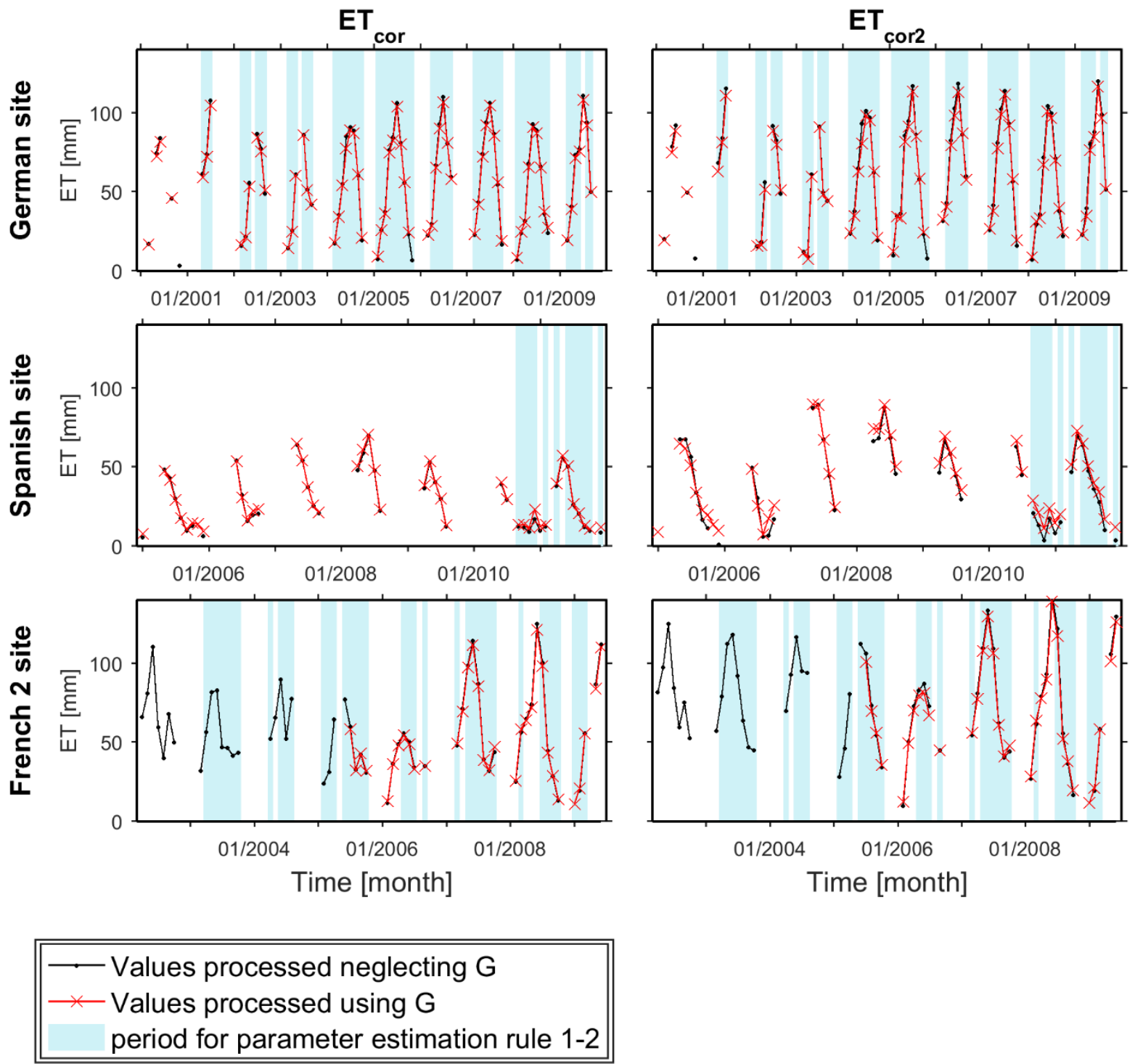

Figure S1. Time series of monthly corrected estimates of actual ET at the German, Spanish and French 2. Reported values were processed for two cases, i.e. neglecting $G$ (ground heat flux) in black and using $G$ in red. The two correct estimates $E_{a c t, c o r}$ and $E_{a c t, c o r 2}$ are defined in Eq. (S1-S2). The blue shaded areas indicate the time period for which ET measurements were used to estimate the model parameters (Sect. 4.1) because the forcing data have a sufficient quality (few gaps) so that we can sensibly compare simulations and observations. 


\section{Analysis of the uncertainty in observed actual ET}

We then analysed the uncertainty in observed ET. We calculated the bias Bias $_{2}$ [\%] and the monthly correlation coefficient $\rho_{2}[-]$ between the uncorrected actual ET estimate and the corrected estimates $E_{\text {act,cor }}$ and $E_{\text {act,cor2 } 2}$ (assessed neglecting the ground heat flux since we have previously shown that it is a reasonable assumption) at the four FLUXNET sites. For instance, for $E_{a c t, c o r}, B_{i a s_{2}}$ was calculated as follows:

$$
\operatorname{Bias}_{2}=100 \frac{\sum_{t \in M_{E T}}\left(E_{a c t, c o r}(t)-E_{a c t, o b s}(t)\right)}{\sum_{t \in M_{E T}} E_{a c t, o b s}(t)}
$$

Where $E_{a c t, o b s}\left[\mathrm{~mm} \mathrm{month}^{-1}\right]$ is the uncorrected observed actual ET equal to $\frac{L E}{\lambda}$.

Figure S2 reports the monthly time series of $E_{a c t, o b s}, E_{a c t, c o r}$ and $E_{a c t, c o r 2}$ and values of Bias $_{2}$ and $\rho_{2}$ are reported in Table S14. We observe that Bias $_{2}$ can be quite large, especially for $E_{\text {act,cor2 }}$, since the relative difference can be as high as $77 \%$ for the French 2 site. We see that $E_{\text {act,cor }}$ provides an intermediate value, between the uncorrected and the residual corrected estimate. The correlation coefficient $\rho_{2}$ was always high at all sites (above 0.86), which means that all three estimated have similar temporal dynamics.

Therefore, the magnitude of observed actual ET has large uncertainties at the FLUXNET sites, while we can have a much higher confidence regarding the temporal dynamics of observed actual ET.

\begin{tabular}{lcccc}
\hline \multirow{2}{*}{ Site } & \multicolumn{3}{c}{ Bias $_{2}[\%]$} & \multicolumn{3}{c}{$\rho_{2}[-]$} \\
\cline { 2 - 5 } & $E_{\text {act,cor }}$ & $E_{\text {act,cor } 2}$ & $E_{\text {act,cor }}$ & $E_{\text {act,cor } 2}$ \\
\hline German & 16 & 23 & 0.97 & 0.94 \\
Spanish & 17 & 76 & 0.99 & 0.87 \\
French 1 & 10 & 30 & 0.97 & 0.91 \\
French 2 & 34 & 77 & 0.97 & 0.86 \\
\hline
\end{tabular}

Table S14. Bias Bias $_{2}$ and monthly correlation coefficient $\rho_{2}$ between monthly measured actual evapotranspiration $\left(E_{a c t, o b s}\right)$ and monthly actual evapotranspiration estimate corrected using the Bowen method $\left(E_{a c t, c o r}\right)$ or the energy residual method $\left(E_{a c t, c o r 2}\right)$ at the four FLUXNET sites. 


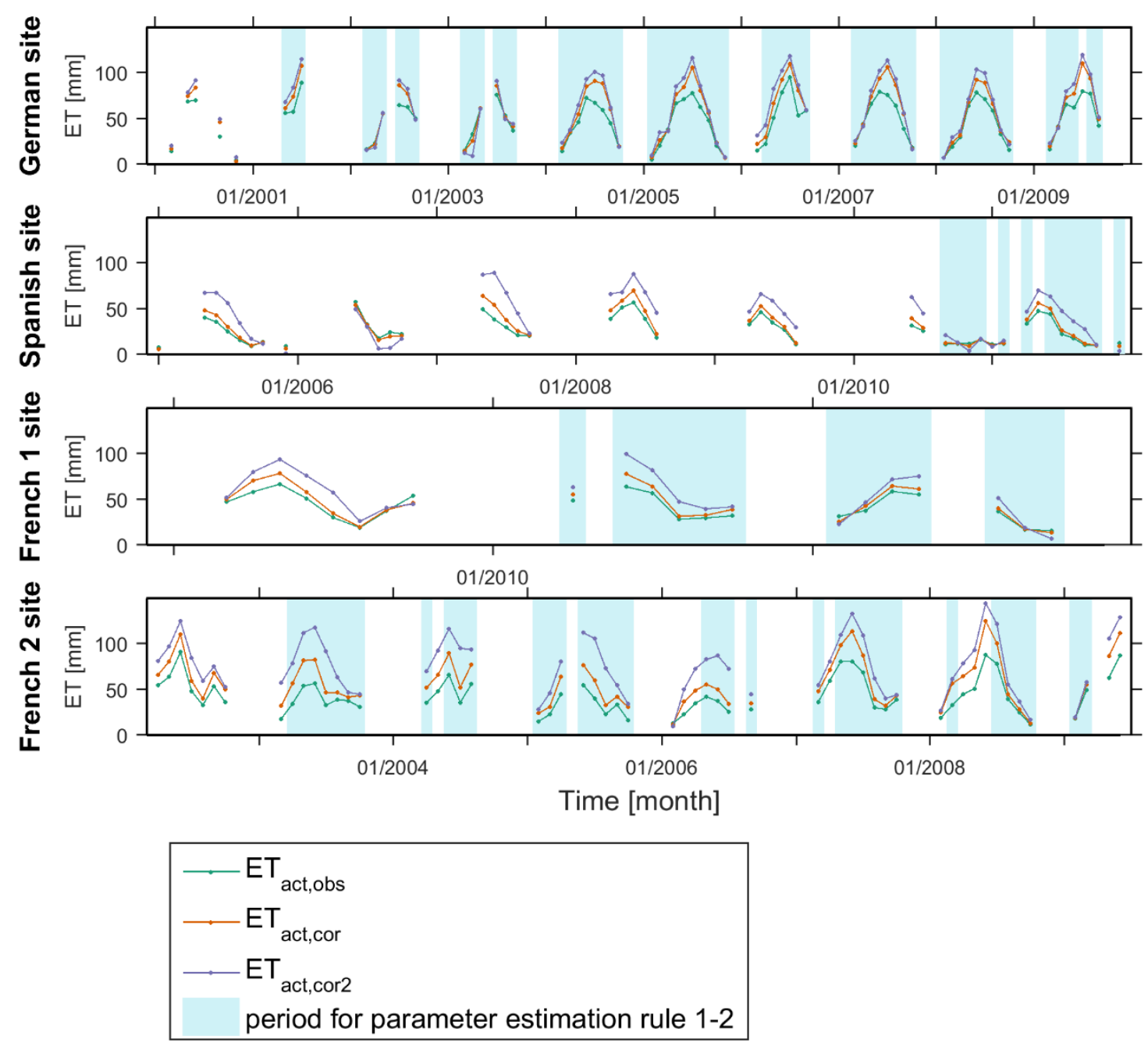

Figure S2. Time series of monthly uncorrected estimate of actual ET ( $\left.E_{\text {act,obs }}\right)$ and corrected estimates $\left(E_{a c t, c o r}\right)$ and $\left(E_{\text {act,cor } 2}\right)$ (Eq. (S1-S2)) for the four FLUXNET sites. The blue shaded areas indicate the time period for which ET measurements were used to estimate the model parameters (Sect. 4.1) because the forcing data have a sufficient quality (few gaps) so that we can sensibly compare simulations and observations

\section{S4.3 Estimation of wind speed at $\mathbf{4 3 . 5} \mathrm{m}$ high at the Spanish site for the virtual experiments}

To set up the virtual experiments, we transformed the wind speed measurements at the Spanish site to estimate their value at the same height as measured at the German site $(43.5 \mathrm{~m})$. In fact, at the Spanish site wind speed is measured at a low height $(2.5 \mathrm{~m})$, since the vegetation is short. Therefore, to simulate the impact of a change to tall vegetation (forest) at the shrub virtual site, wind speed should be estimated at a height which is above canopy level, as required by the Penman Monteith equation. We assumed a logarithmic wind profile as e.g. in Lhomme et al. (2014). We note that we modified Eq. (6) in Lhomme et al. (2014), which is valid when the vegetation is fully covering the ground, to account for sparse vegetation. We calculated the value of wind speed at $43.5 \mathrm{~m}$ over vegetated and non-vegetated fraction separately using Eq. (6) in Lhomme et al. (2014) and we estimated the overall wind speed at $43.5 \mathrm{~m}$ for the site as the area weighted value over both fractions. The other weather variables (air temperature and humidity) are assumed to be the same at $43.5 \mathrm{~m}$ and $2.5 \mathrm{~m}$. We deemed that these assumptions were reasonable, since the objective of the virtual experiments is to understand recharge sensitivity and not to predict recharge. 


\section{S5. Analysis of the impact of the warm-up period on predictions at FLUXNET sites}

The analyses reported in this section aim to identify an appropriate value of the warm-up period (denoted as $H_{w}[$ month] $)$, to evaluate V2Karst at the four FLUXNET sites. The warm-up period corresponds to the initial time period which is discarded to reduce the impact of the choice of the value of the model initial states on the simulations.

We assessed the sensitivity of the fluxes simulated with V2Karst to $H_{w}$ by running the model for a range of values of $H_{w}$. For a given FLUXNET site, the date of the first day following the warm-up period is kept constant across the simulations (1 January 2001 at the German site, 1 January 2006 at the Spanish site, 1 January 2010 at the French 1 site and 1 April 2003 at the French 2 site). Instead, the date of the first day of the warm-up period is varied following the value of $H_{w}$. In this way, simulated fluxes are assessed over the same time horizon for all values of $H_{w}$ and therefore simulations using different values of $H_{w}$ can be compared among each other. We varied $H_{w}$ between 2 and 12 months and we assessed the sensitivity of the total simulated recharge $\left(Q_{e p i}\right)$ and actual ET $\left(E_{a c t}\right)$ to $H_{w}$, because we are interested in these two variables in our study. We estimated the metrics $\Delta Q_{e p i}[\mathrm{~mm}]$ and $\Delta E_{\text {act }}[\mathrm{mm}]$ defined as follows:

$$
\begin{gathered}
\Delta Q_{e p i}\left(H_{w}=h_{w}\right)=Q_{e p i}\left(H_{w}=h_{w}\right)-Q_{e p i}\left(H_{w}=12\right) \\
\Delta E_{a c t}\left(H_{w}=h_{w}\right)=E_{a c t}\left(H_{w}=h_{w}\right)-E_{a c t}\left(H_{w}=12\right)
\end{gathered}
$$

where $h_{w}=2, \ldots, 11$ months

15 The two metrics of Eq. (S5) measure the difference in $Q_{\text {epi }}$ and $E_{a c t}$ when $H_{w}$ is set to 12 months compared to when $H_{w}$ is set to lower values. A large value of $\Delta Q_{e p i}$ or $\Delta E_{a c t}$ means that the choice of $H_{w}$ has an impact on simulated recharge and actual ET, while a small value of $\Delta Q_{e p i}$ or $\Delta E_{a c t}$ means that $H_{w}$ has little effect on the simulation results. Initially, we assumed that the soil and epikarst stores for all model vertical compartments of V2Karst are saturated. For each of the 11 values of $H_{w}$ that were tested, we repeated the simulations over 1,000 parameter sets sampled using latin hypercube sampling and the ranges reported in Table 1 of the main paper. Therefore, for each site, we performed a total number of 11,000 model executions.

Figure S3 reports $\Delta Q_{e p i}$ (left panels) and $\Delta E_{a c t}$ (right panels) against $H_{w}$ for the 1,000 parameter sets for each FLUXNET site. We see that when $H_{w}$ increases, the width of the simulation ensemble decreases, which means that the impact of $H_{w}$ on the simulations decreases. In general, the value of $\Delta Q_{e p i}$ and $\Delta E_{\text {act }}$ becomes very small $\left(-5 \mathrm{~mm}<\Delta Q_{e p i}<5 \mathrm{~mm}\right.$ and $-5 \mathrm{~mm}<\Delta E_{a c t}<5 \mathrm{~mm}$ ) when $H_{w}$ is equal to or larger than 10 months, apart from one parameterisation at the Spanish site for which $\Delta Q_{e p i}$ and $\Delta E_{a c t}$ becomes very small when $H_{w}$ is equal to 11 months. Therefore, the simulated fluxes show generally little changes in response to changes in $H_{w}$ when $H_{w}$ is higher than 10 months.

Consequently, we deemed reasonable to set the warm-up period equal to 12 months at all FLUXNET sites to perform the parameter estimation and the sensitivity analysis in this study (Sect. 4.1 and 4.2). 

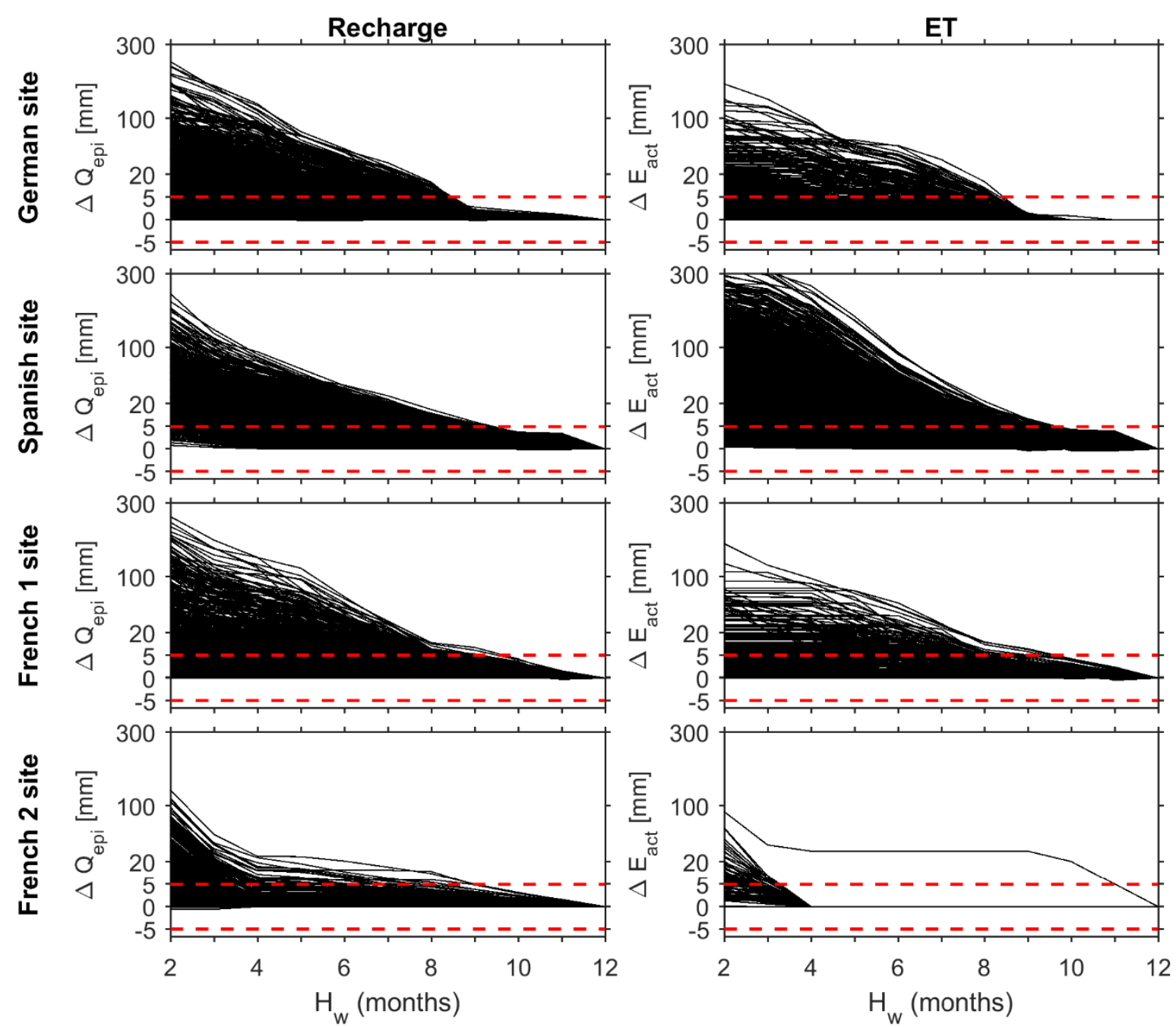

Figure S3. Difference in simulated recharge $\Delta Q_{\text {epi }}$ and actual ET $\Delta E_{\text {act }}$ (defined in Eq. (S5)) against the length warm-up period $\left(H_{w}\right)$. 


\section{S6. Analysis of the range of variation of the precipitation characteristics to inform the setup of the virtual experiments}

This section aims to inform the choice of the ranges of the monthly precipitation $P_{m}\left[\mathrm{~mm}\right.$ month $\left.{ }^{-1}\right]$, the precipitation intensity $I_{p}\left[\mathrm{~mm} \mathrm{~d}^{-1}\right]$ and the interval between rainy days $H_{p}[\mathrm{~d}]$ to derive the synthetic

5 precipitation inputs used in the virtual experiment (Sect. 4.3). This section reports the cumulative distribution function of $P_{m}$ (Fig. S4), $I_{p}$ (Fig. S5) and $H_{p}$ (Fig. S6) for:

- the whole domain, which is all European and Mediterranean carbonate rock areas reported in the carbonate rock map of Williams and Ford (2006) presented in Fig.1 in the main paper. For this, precipitation from the GLDAS database is used (Rodell et al., 2004);

10 - the four carbonate rock sites of the FLUXNET network (Baldocchi et al., 2001) analysed in this study and presented in Fig. 1 and Table B1. 

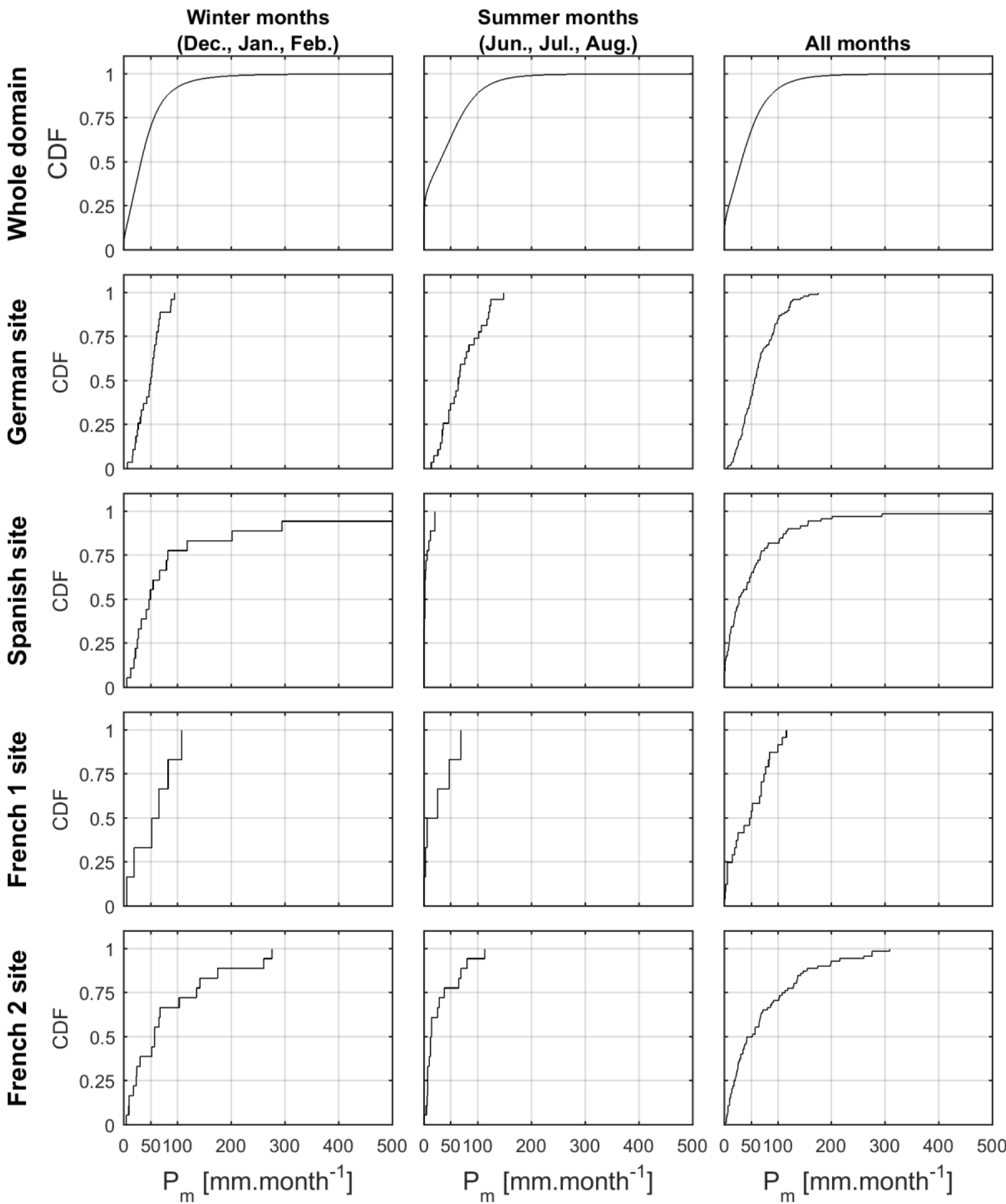

Figure S4. Cumulative distribution function of monthly precipitation $P_{m}\left[\mathrm{~mm}\right.$ month $\left.^{-1}\right]$ over winter months (Dec., Jan. Feb.), summer months (Jun., Jul., Aug.) and all months of the year estimated for the whole domain (all European and Mediterranean carbonate rock areas) over the period 1 October 2002-30 September 2012, at the German FLUXNET site over the period 1 January 2001-17 December 2009, at the Spanish FLUXNET site over the period 1 January 2006-30 December 2011, at the French 1 FLUXNET site over the period 1 January 2010-30 December 2011 and at the French 2 FLUXNET site over the period 1 April 2003-31 March 2009. 

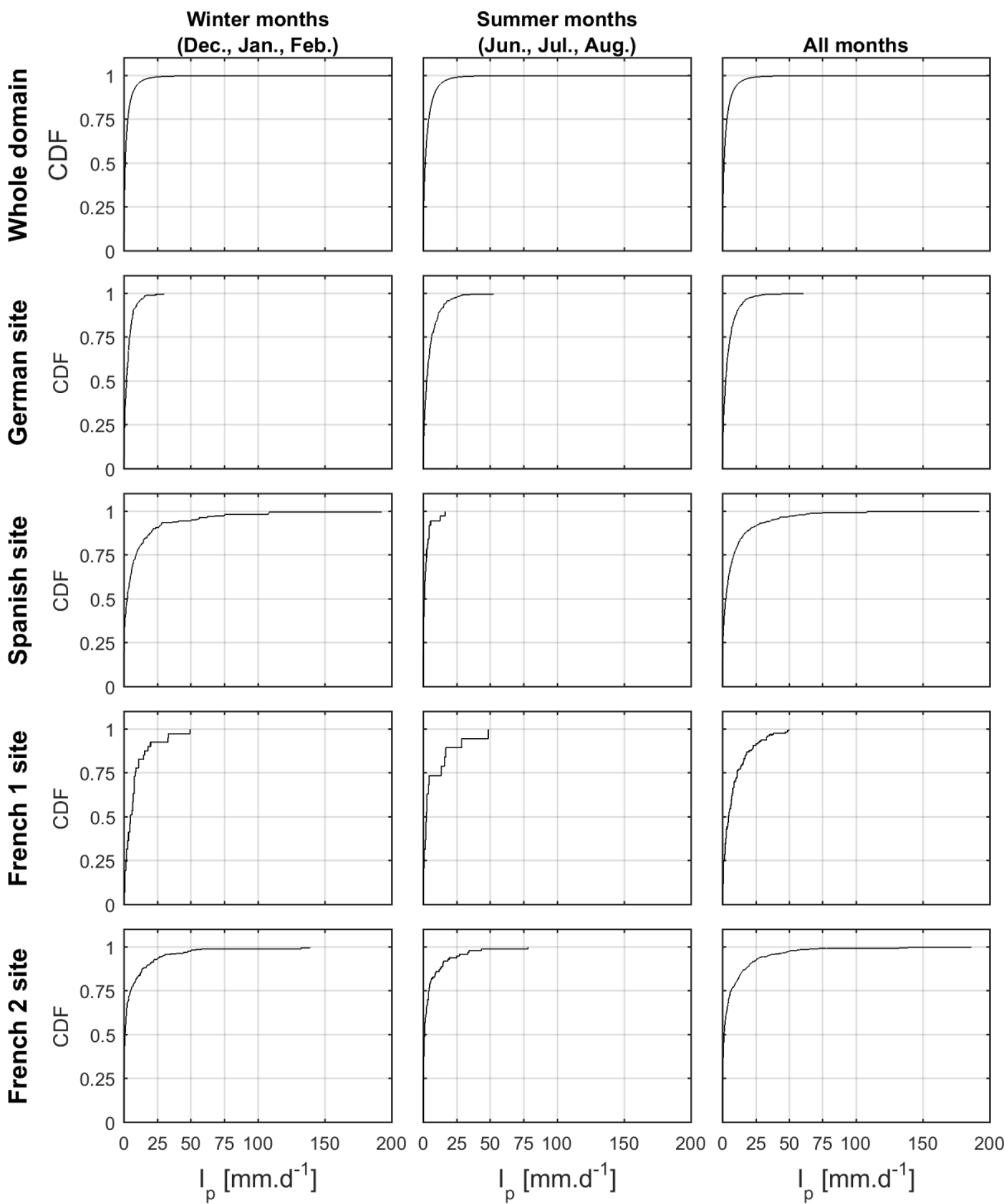

Figure S5. Cumulative distribution function of the precipitation intensity $I_{p}\left[\mathrm{~mm} \mathrm{~d}^{-1}\right]$ over winter months (Dec., Jan. Feb.), summer months (Jun., Jul., Aug.) and all months of the year estimated for the whole domain (all European and Mediterranean carbonate rock areas) over the period 1 October 2002-30 September 2012, at the German FLUXNET site over the period 1 January 2001-17 December 2009, at the Spanish FLUXNET site over the period 1 January 2006-30 December 2011, at the French 1 FLUXNET site over the period 1 January 2010-30 December 2011 and at the French 2 FLUXNET site over the period 1 April 2003-31 March 2009. Only days that had a precipitation amount above $0.1 \mathrm{~mm}$ were included in the calculation. 

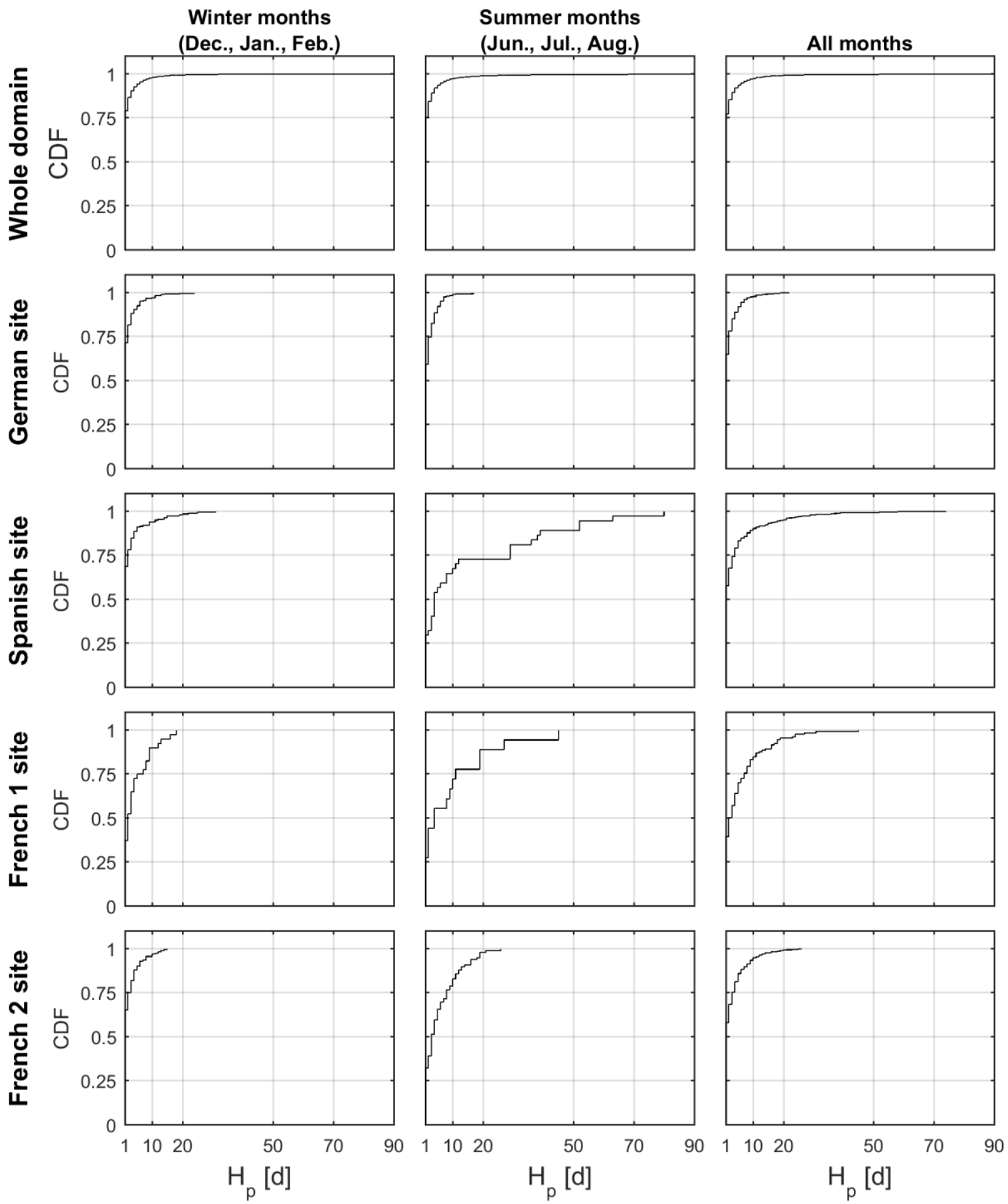

Figure S6. Cumulative distribution function of the interval between wet days $H_{p}[d]$ over winter months (Dec., Jan. Feb.), summer months (Jun., Jul., Aug.) and all months of the year estimated for the whole domain (all European and Mediterranean carbonate rock areas) over the period 1 October 2002-30 September 2012, at the German FLUXNET site over the period 1 January 2001-17 December 2009, at the Spanish FLUXNET site over the period 1 January 2006-30 December 2011, at the French 1 FLUXNET site over the period 1 January 2010-30 December 2011 and at the French 2 FLUXNET site over the period 1 April 2003-31 March 2009. A wet day is defined as a day with more than $0.1 \mathrm{~mm}$ of precipitation. 


\section{S7. Global sensitivity analysis of V2Karst parameters for the standard deviation of monthly simulated recharge and for simulated actual transpiration}

This section reports additional results for the global sensitivity analysis of the V2Karst parameters (Sect. 4.2). While in Sect. 5.2 and Fig. 7 we present the results for total recharge, here Fig. S7 and S8 report the results for the standard deviation of monthly recharge and for actual transpiration respectively. Figure 7 shows that some parameters that have a very small effect on total recharge at all sites and for both range choices (left and right panels in Fig. 7). The additional sensitivity analyses presented in this section reveal that some of these parameters have an influence on other aspects of the model simulations.

For example, we find that parameters $K_{e p i}$ and $V_{\text {epi }}$ have a small impact on total recharge $\left(\mu^{*}<3 \%\right.$ in all

10 plots in Fig. 7), while they have an effect on the standard deviation of the recharge (Fig. S7). The same holds for parameter $f_{\text {red }}$, which also has a small impact on total recharge but a significantly higher importance on the standard deviation of the recharge, in particular at the Spanish and French 1 site (Fig. 7). Given that the standard deviation of recharge is a proxy metric for recharge dynamics, we infer that parameters $K_{e p i}, V_{e p i}$ and $f_{\text {red }}$ have a significant effect on how recharge is distributed in time, but a limited effect on its total amount.

15 Furthermore, parameters $V_{e}$ and $k$ have little effect on total recharge ( $\mu^{*}<3 \%$ in all plots in Fig. 7), while they are influential with respect to the percentage of actual transpiration in total ET (Fig. S8). Therefore, both parameters have an impact on the partitioning of ET among its three components. 

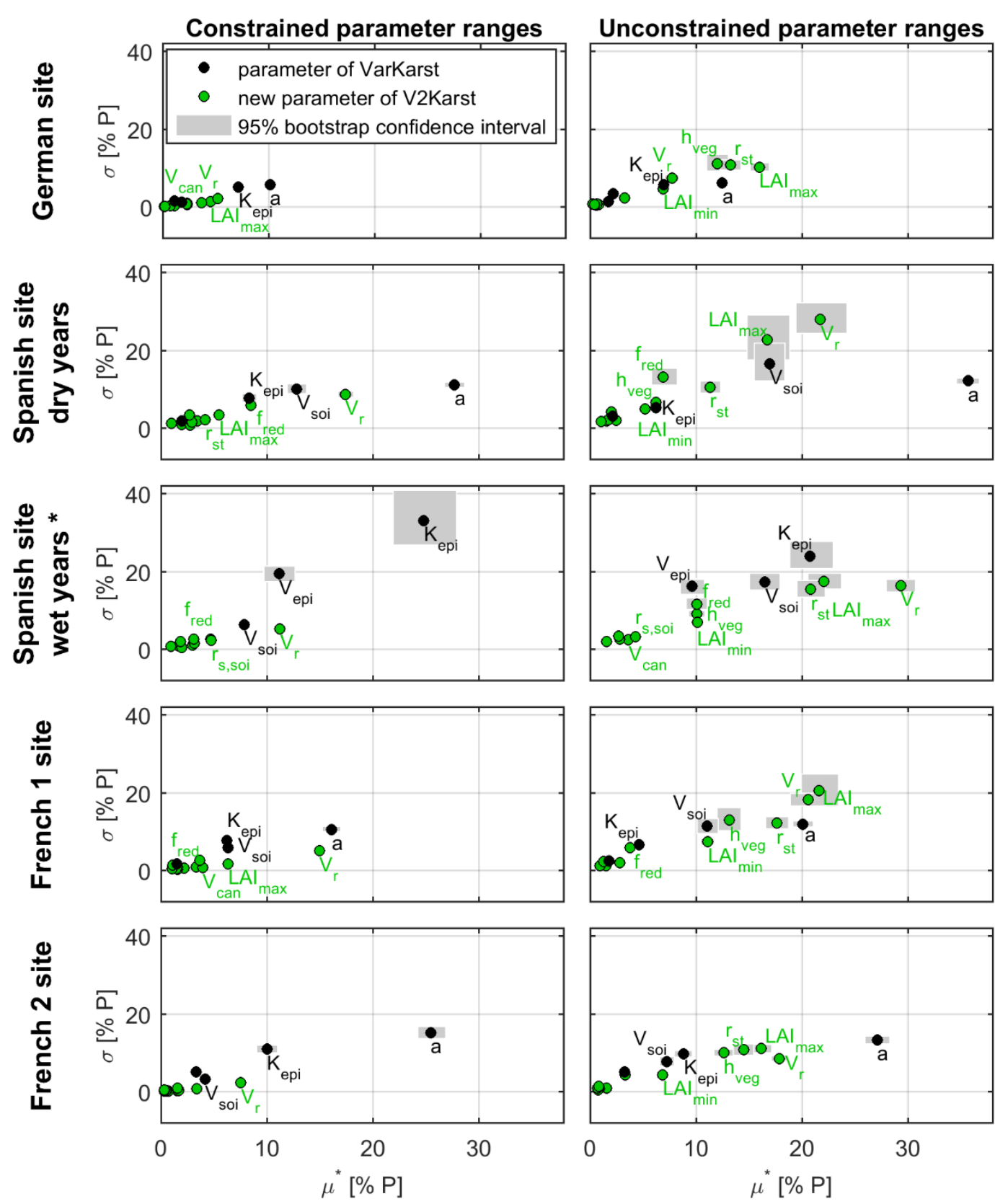

Figure S7. Sensitivity indices of the V2Karst parameters $\left(\mu^{*}\right.$ is the mean of the absolute Elementary Effects and $\sigma$ is the standard deviation of the Elementary Effects) for the standard deviation of simulated monthly recharge (expressed as a percentage of mean monthly precipitation) at the four FLUXNET sites when constrained (site-specific) parameter ranges are used (ranges of Table 3 in the main paper) and when unconstrained ranges are used (ranges of Table 1 in the main paper). Sensitivity indices were computed over the period 1 January 2001-17 December 2009 for the German site, 1 January 2006-31 December 2008 for the Spanish site (dry years), 1 January 2009-30 December 2011 for the Spanish site (wet years), 1 January 201030 December 2011 for the French 1 site and 1 April 2003-31 March 2009 for the French 2 site.* Sensitivity indices for parameter $a$ are not reported in the plots for the Spanish site wet years because they are significantly higher than the other parameters ( $\mu_{a}^{*}=68 \%$ and $\sigma_{a}=51 \%$ for constrained ranges and $\mu_{a}^{*}=68 \%$ and $\sigma_{a}=38 \%$ for unconstrained ranges). 

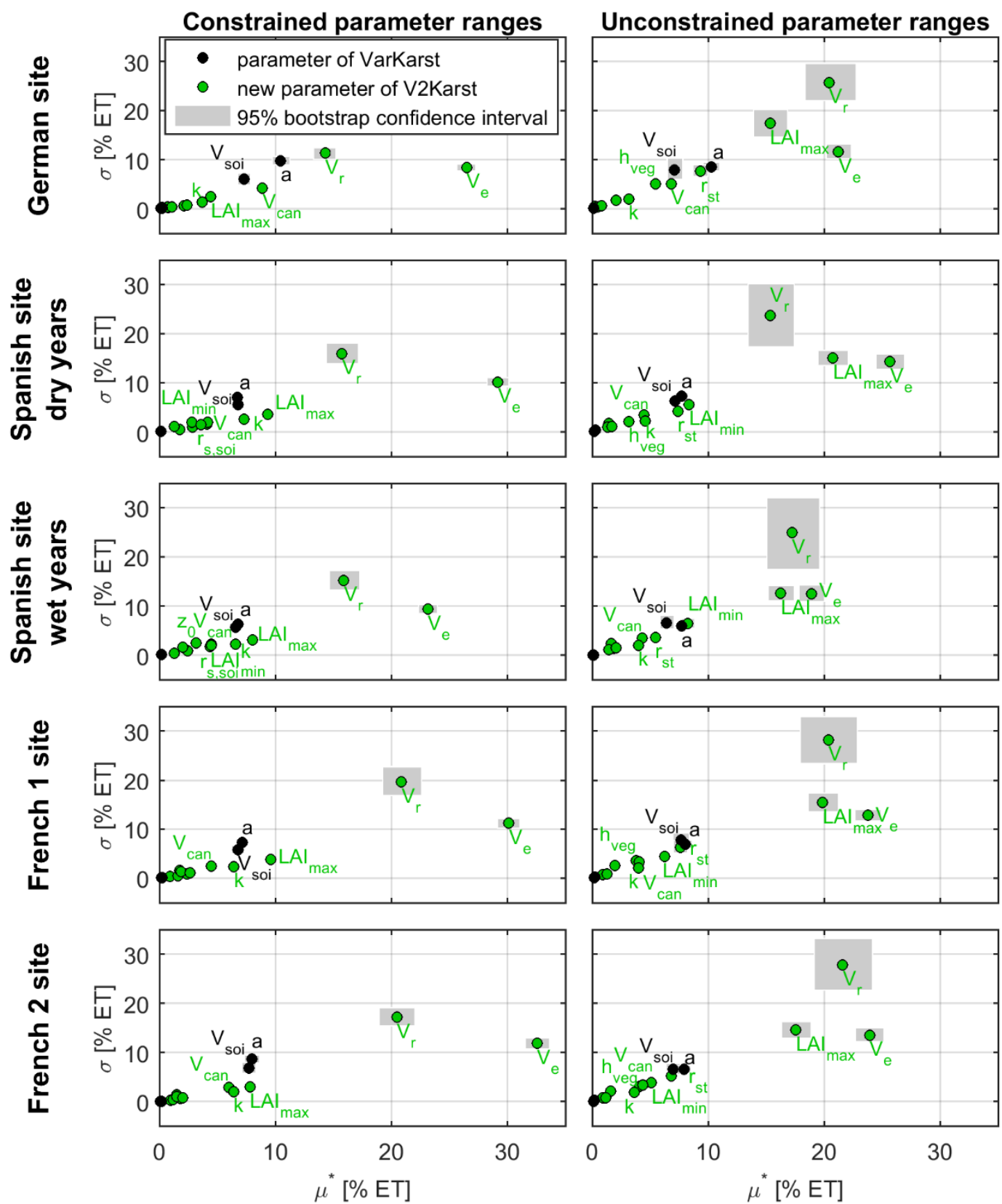

Figure S8. Sensitivity indices of the V2Karst parameters $\left(\mu^{*}\right.$ is the mean of the absolute Elementary Effects and $\sigma$ is the standard deviation of the Elementary Effects) for simulated actual transpiration (expressed as a percentage of total ET) at the four FLUXNET sites when constrained (site-specific) parameter ranges are used (ranges of Table 3 in the main paper) and when unconstrained ranges are used (ranges of Table 1 in the main paper). Sensitivity indices were computed over the period 1 January 2001-17 December 2009 for the German site, 1 January 2006-31 December 2008 for the Spanish site (dry years), 1 January 2009-30 December 2011 for the Spanish site (wet years), 1 January 2010-30 December 2011 for the French 1 site and 1 April 2003-31 March 2009 for the French 2 site. 


\section{S8. Comparison between V2Karst results obtained using daily and hourly simulation time step}

The V2Karst model (version V1.1) can be run at both daily and sub-daily time step. This section presents a comparison of simulation results obtained using a daily and an hour time step (Fig. S9-S17). We tested the model predictions by estimating the model parameters using the soft rules presented in Sect. 4.1 for both daily and hourly time step. We did not observe significant differences between the results for daily and hourly time step in terms of parameter constraining (Fig. S9-S12) and in terms of model predictions (Fig. S13-S17). This means that the simulation time step has little effect on recharge at monthly and annual time scale, which is the focus of our study. Therefore, it is reasonable to apply the model at daily time step for our application, which significantly reduces the computational requirements.

\section{Description of the experiment setup}

For hourly simulations, we forced V2Karst using measurements of $P, T, R_{n}, R H, W S$ and $G$ at the carbonate rock FLUXNET sites, while $G$ was neglected for daily simulations (as explained in Sect. 2.3.3). We did not run the model at hourly time step at the French 1 site since no measurements of $G$ are available. Forcing and calibration data were processed as explained in Sect. 3.2, S4.1 and S4.2

Table S15 reports the simulation period and the number of monthly latent heat flux and soil moisture observations that were used to estimate the model parameters at the three FLUXNET sites. We note that the simulation period for the French 2 site is reduced compared to the simulation period used to perform the daily analyses presented in the main paper (Table 2). This is because the time series of $G$ contained many gaps for this site and we therefore had to discard part of it to perform hourly simulations.

We run V2Karst against the same sample of the model parameter of size 100,000 and within the ranges of Tables 1 and 3, for both hourly and daily time step (as explained in Sect. 4.1). We note that we applied a conversion factor to parameter $K_{e p i}$ for hourly simulation compared to daily simulations to run the model ( $K_{\text {epi }}$ is expressed in hours for hourly simulations, while it is expressed in days for daily simulations). All model runs were performed using a 1-year warmup period, which we found to be sufficient to remove the impact of the initial conditions on the simulation results in the case of daily time step (Sect. S5). We applied the soft rules to both hourly and daily simulation results, as explained in Sect. 4.1. 


\begin{tabular}{llllc}
\hline Site & \multicolumn{2}{l}{$\begin{array}{l}\text { Simulation period (including a } \\
\text { one-year warm-up period) }\end{array}$} & $\begin{array}{l}\text { Number of months with latent } \\
\text { heat flux measurement for } \\
\text { calibration }\end{array}$ & $\begin{array}{l}\text { Number of months with soil moisture } \\
\text { measurement for calibration }\end{array}$ \\
\cline { 2 - 5 } & Start & End & 62 & 74 \\
\hline German site & 1 Jan. 2000 & 17 Dec. 2009 & 12 & 12 \\
Spanish site & 1 Jan. 2005 & 30 Dec. 2011 & 16 & Not measured \\
French 2 site & 17 Jul. 2005 & 29 Jun. 2009 &
\end{tabular}

Table S15. Simulation period at the three FLUXNET sites, and number of months where latent heat flux measurements and soil moisture measurements are available to calibrate the model. Soil moisture measurements is not provided at the French 2 site.
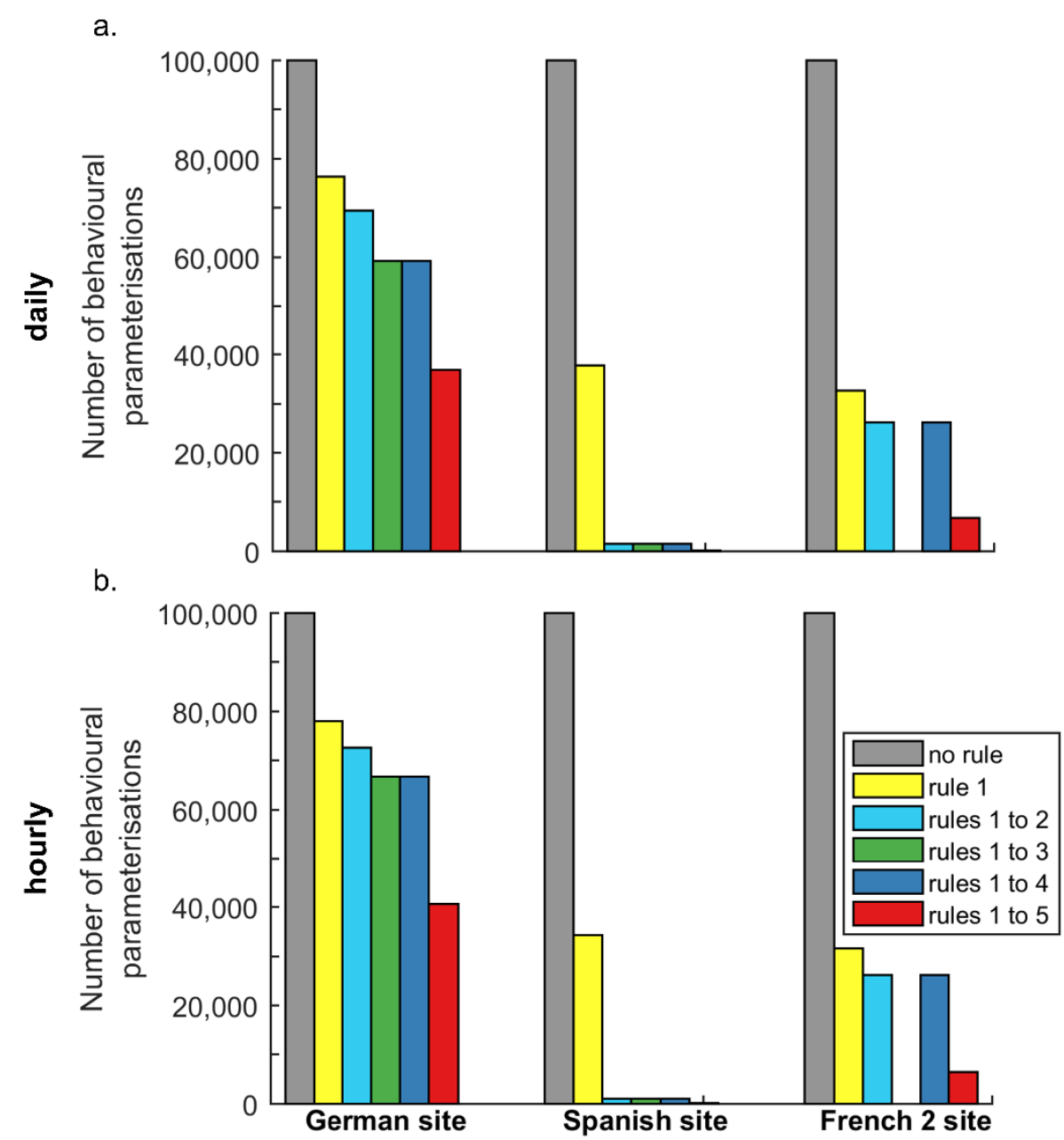

5 Figure S9. Reduction in the number of behavioural parameterisations of the V2Karst model at FLUXNET sites when applying sequentially the five soft rules defined in Sect. 4.1 (no rule: initial sample; rule 1: ET bias; rule 2: ET correlation; rule 3: soil moisture correlation; rule 4: runoff; rule 5: a priori information). Rule 3 could not be applied to the French 2 site where soil moisture observations are not available. (a) results for daily time step and (b) results for hourly time step. 


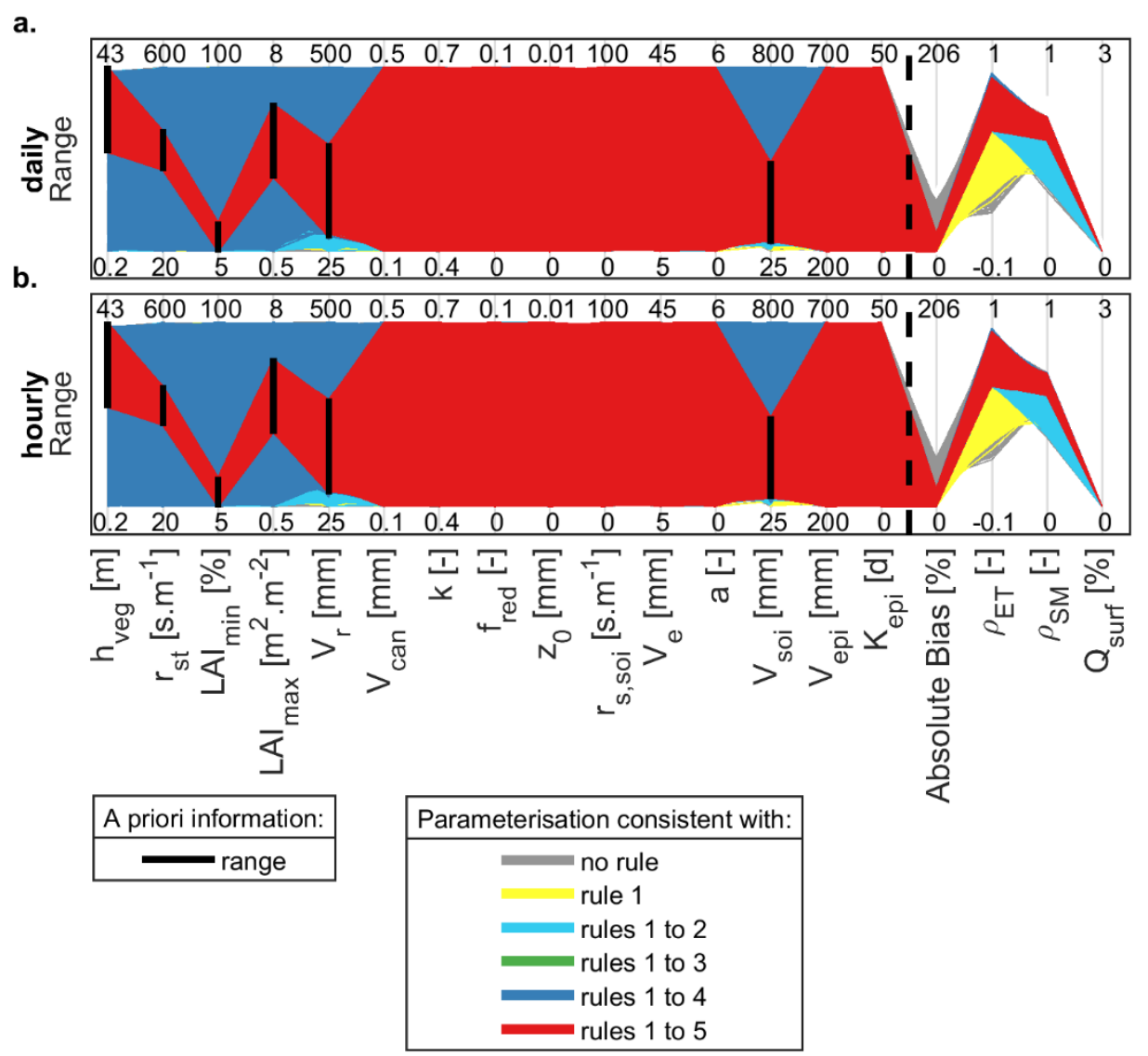

Figure S10. Parallel coordinate plots representing V2Karst behavioural parameterisations and their corresponding simulated output values, identified when sequentially applying the five soft rules defined in Sect. 4.1 at the German site for (a) daily time step and (b) hourly time step. Parameters are defined in Tables 1 and S11. BIAS absolute mean error between observed and simulated total actual ET (rule 1), $\rho_{E T}$ correlation coefficient between observed and simulated total actual ET (rule 2), $\rho_{S M}$ correlation coefficient between observed and simulated soil moisture (rule 3), $Q_{\text {surf }}$ surface runoff (rule 4). Rule 5 corresponds to application of a priori information on parameter ranges (black vertical bars, Tables 3 and S12). 


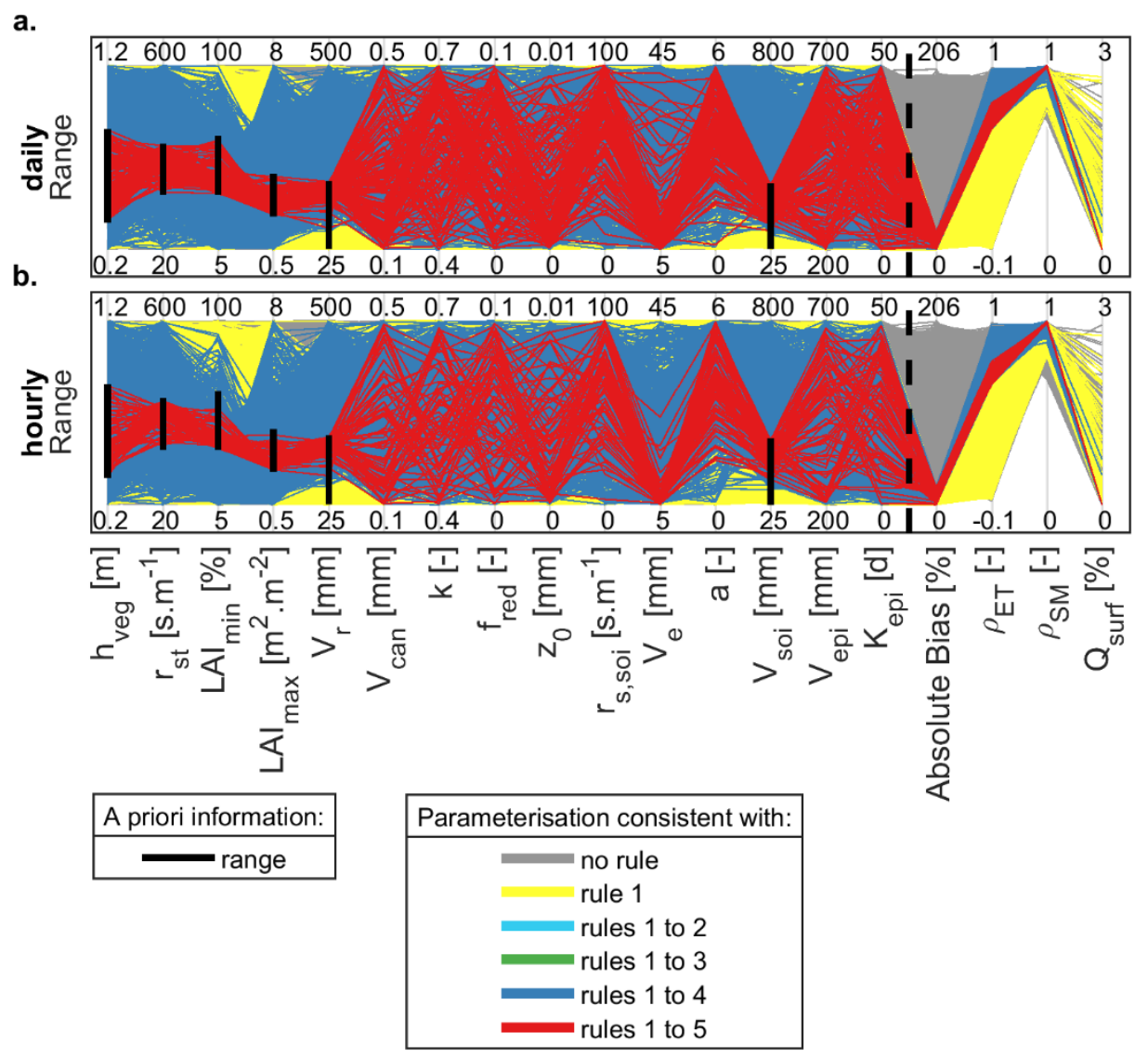

Figure S11. Parallel coordinate plots representing V2Karst behavioural parameterisations and their corresponding simulated output values, identified when sequentially applying the five soft rules defined in Sect. 4.1 at the Spanish site for (a) daily time step and (b) hourly time step. Parameters are defined in Table 1. BIAS absolute mean error between observed and simulated total actual ET (rule 1), $\rho_{E T}$ correlation coefficient between observed and simulated total actual ET (rule 2), $\rho_{S M}$ correlation coefficient between observed and simulated soil moisture (rule 3), $Q_{\text {surf }}$ surface runoff (rule 4). Rule 5 corresponds to application of a priori information on parameter ranges (black vertical bars, Tables 3 and S12). 


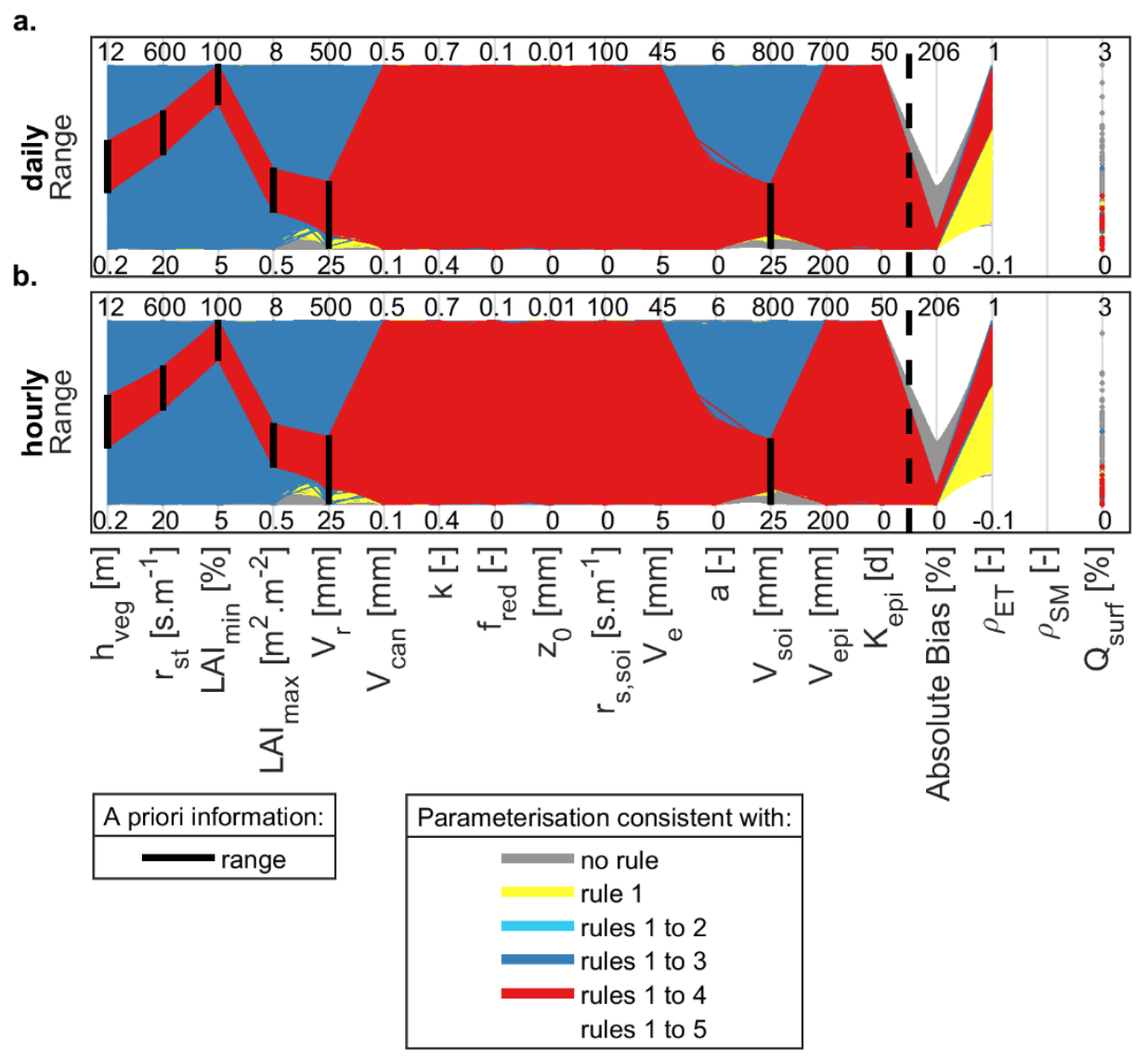

Figure S12. Parallel coordinate plots representing V2Karst behavioural parameterisations and their corresponding simulated output values, identified when sequentially applying the five soft rules defined in Sect. 4.1 at the French 2 site for (a) daily time step and (b) hourly time step. Parameters are defined in Tables

51 and S11. BIAS absolute mean error between observed and simulated total actual ET (rule 1), $\rho_{E T}$ correlation coefficient between observed and simulated total actual ET (rule 2), $\rho_{S M}$ correlation coefficient between observed and simulated soil moisture (rule 3 ), $Q_{\text {surf }}$ surface runoff (rule 4). Rule 5 corresponds to application of a priori information on parameter ranges (black vertical bars, Tables 3 and S12). 

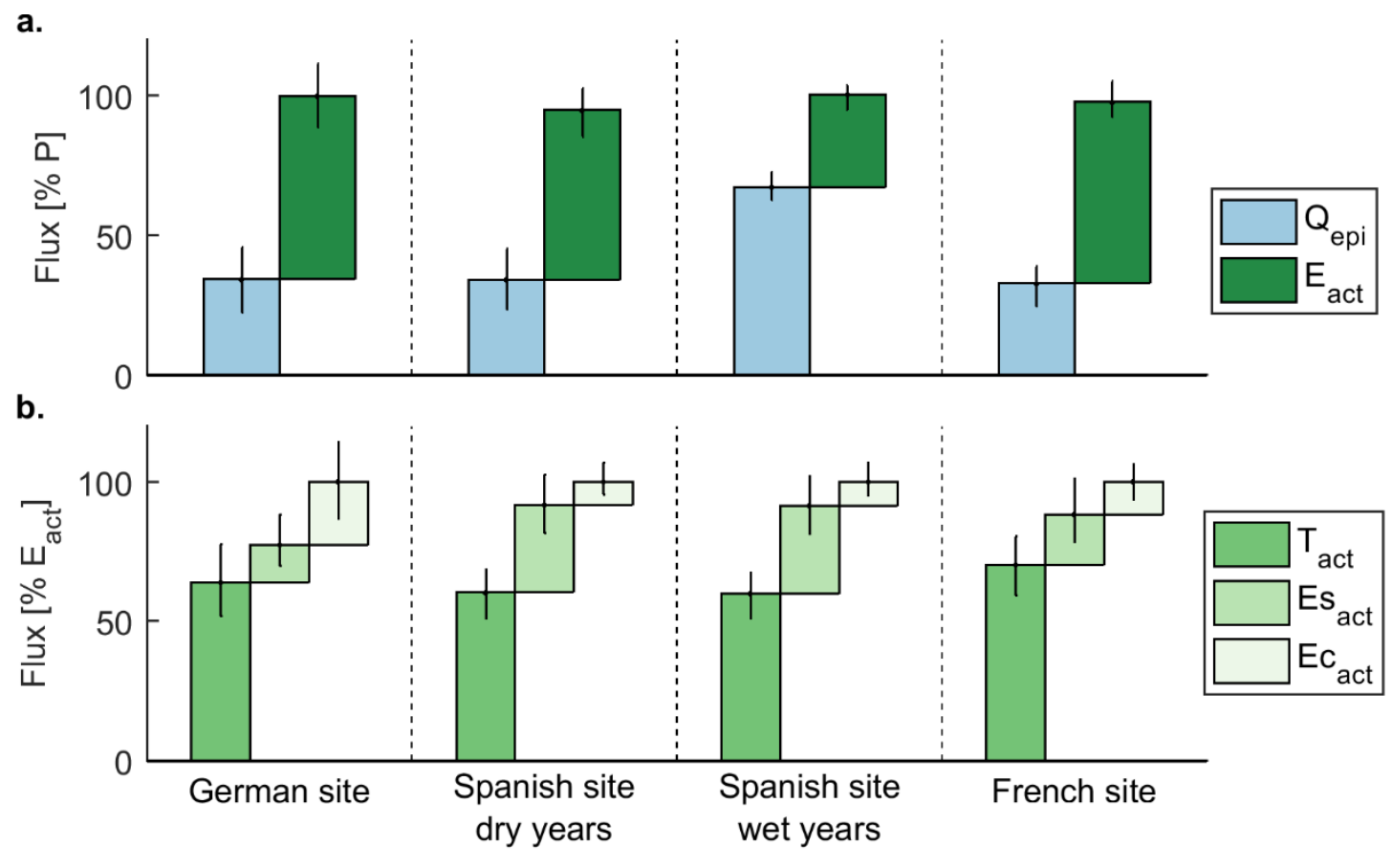

Figure S13. Model outputs assessed using a daily time step: (a) Simulated recharge $\left(Q_{\text {epi }}\right)$ and actual ET $\left(E_{a c t}\right)$ expressed as a percentage of total precipitation and (b) simulated actual transpiration $\left(T_{a c t}\right)$, actual soil evaporation $\left(E s_{a c t}\right)$ and actual evaporation from interception $\left(E c_{a c t}\right)$ expressed as a percentage of $E_{\text {act }}$. The figure reports the ensemble mean and $95 \%$ confidence intervals calculated over the behavioural simulation ensemble of the V2Karst model at the four FLUXNET sites. Simulated fluxes were evaluated over the period 1 January 2001-17 December 2009 for the German site, 1 January 2006-31 December 2008 for the Spanish site (dry years), 1 January 2009-30 December 2011 for the Spanish site (wet years), 1 January 2010-30 December 2011 for the French 1 site and 1 April 2003-31 March 2009 for the French 2 site. Mean annual water balance for behavioural set.

a.

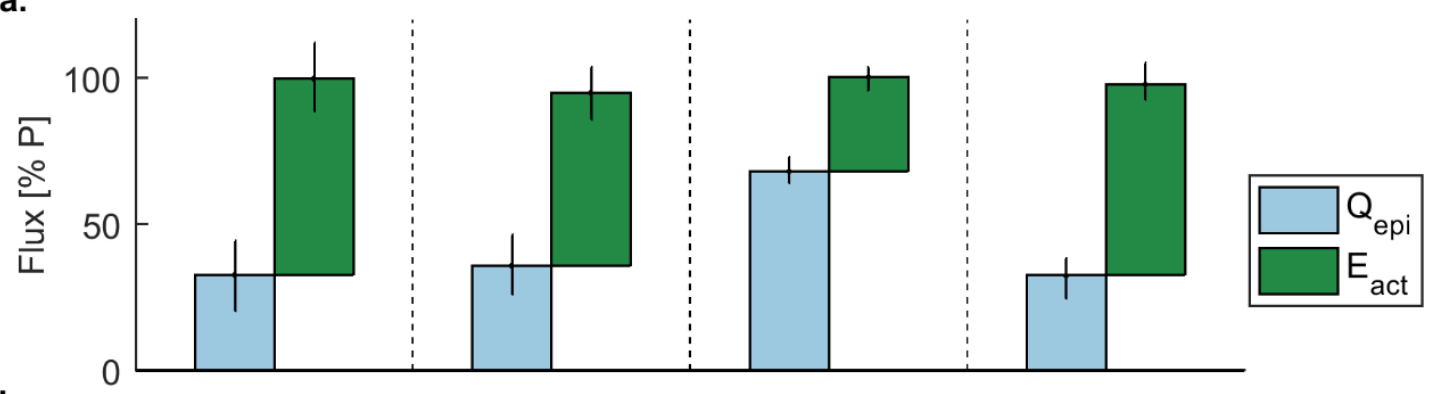

b.

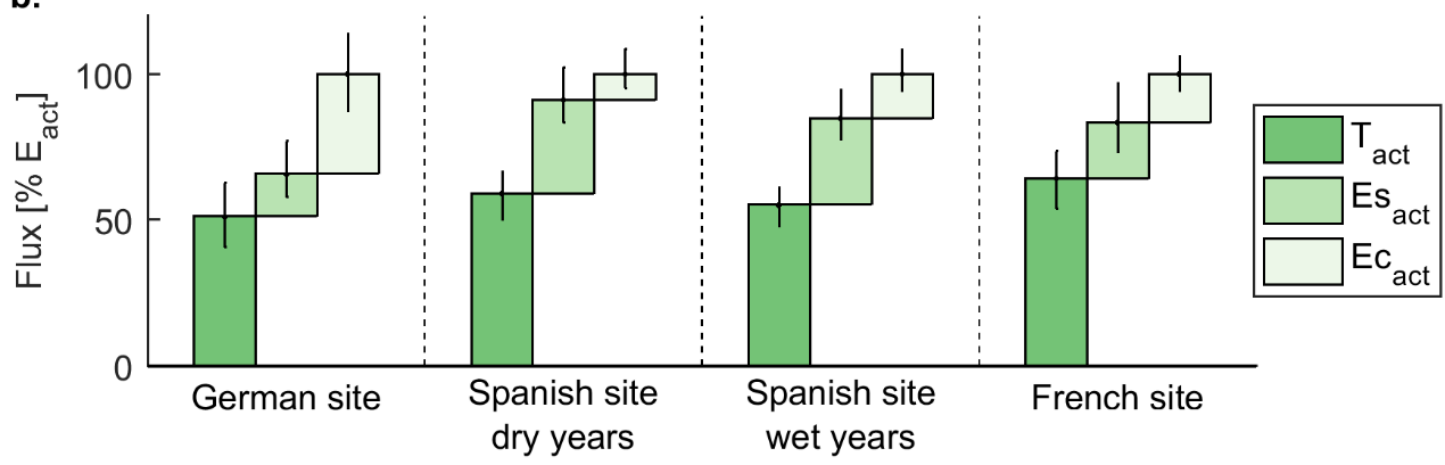

Figure S14. Same as Figure S13 but using an hourly time step. 
a. daily

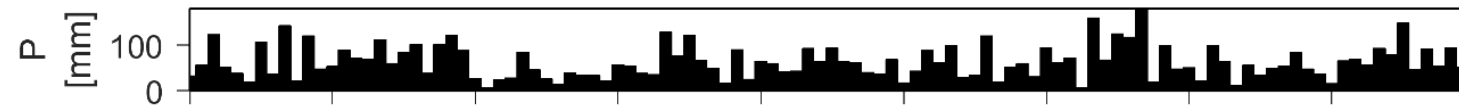
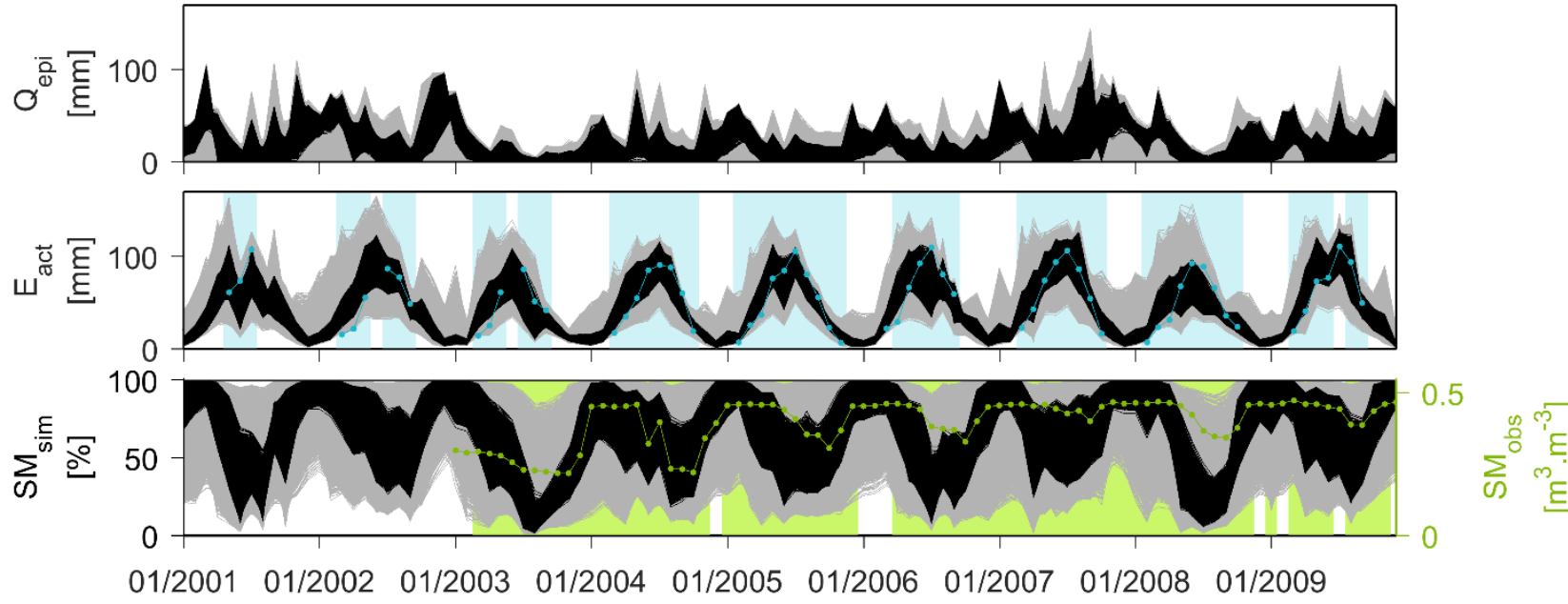

b. hourly

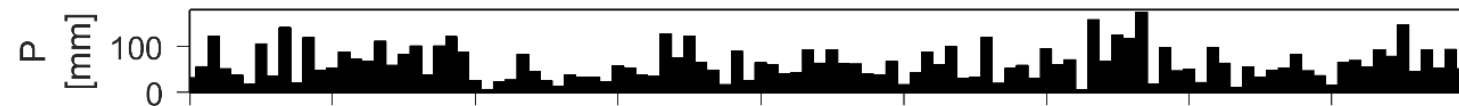
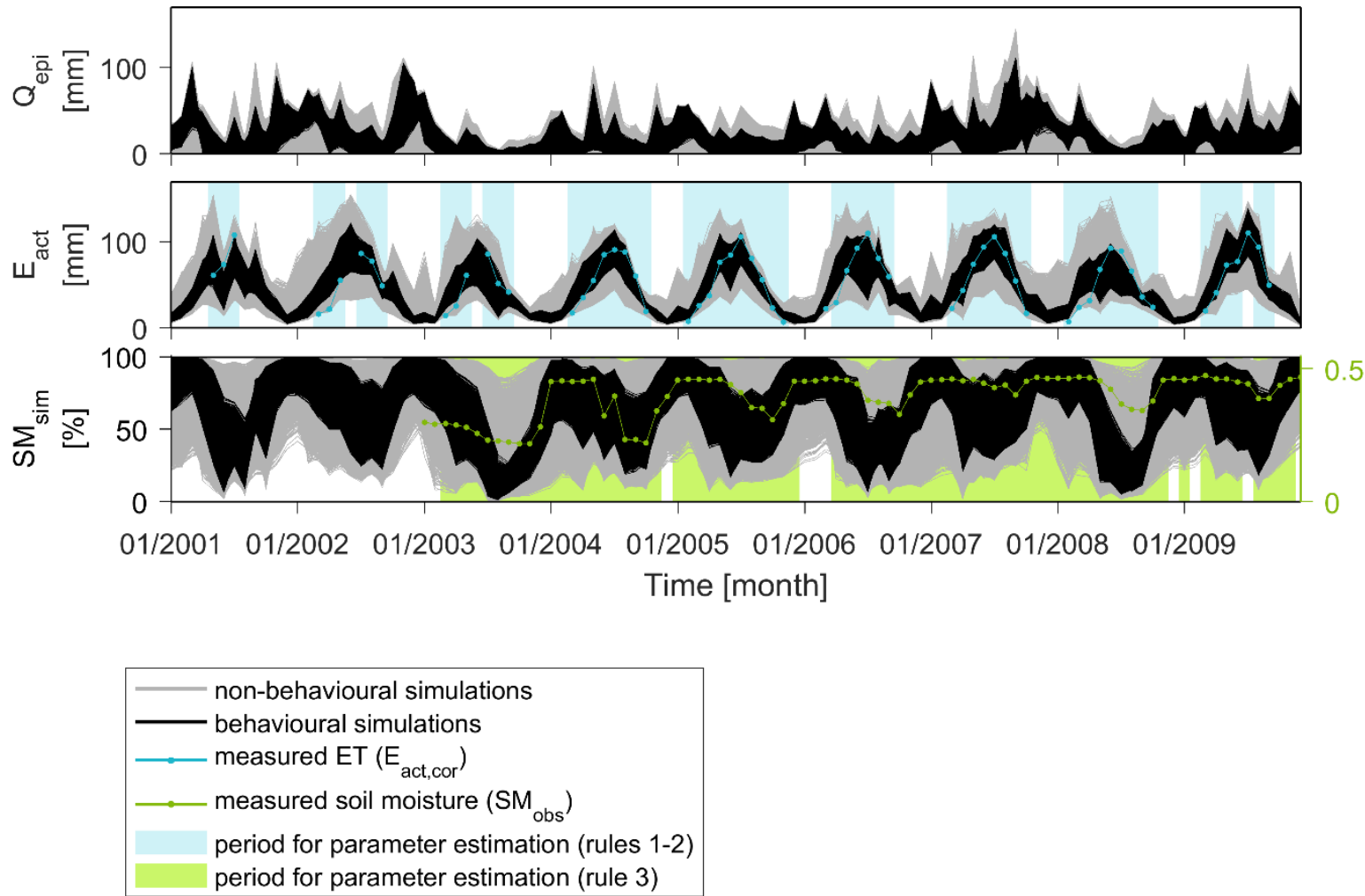

Figure S15. Monthly time series of precipitation input $(P)$, simulated recharge $\left(Q_{\text {epi }}\right)$, simulated actual ET ( $E_{a c t}$, which is the sum of evaporation from canopy interception, transpiration and soil evaporation), simulated soil moisture within the root zone $\left(S M_{\text {sim }}\right)$, and monthly observations of actual ET and soil moisture at the

5 German site for (a) daily time step, (b) hourly time step. Blue and green shaded areas correspond to the periods in which observation of ET and soil moisture respectively were selected to apply the soft rules of Sect. 4.1 (further details on data processing in Sect. 3.2, S4.1 and S4.2). 


\section{a. daily}

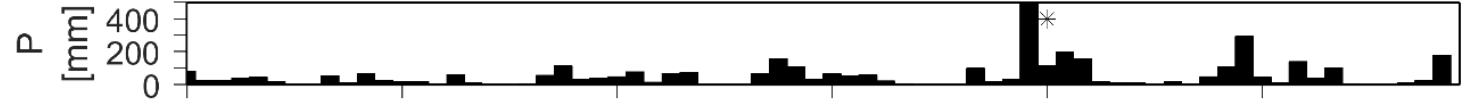
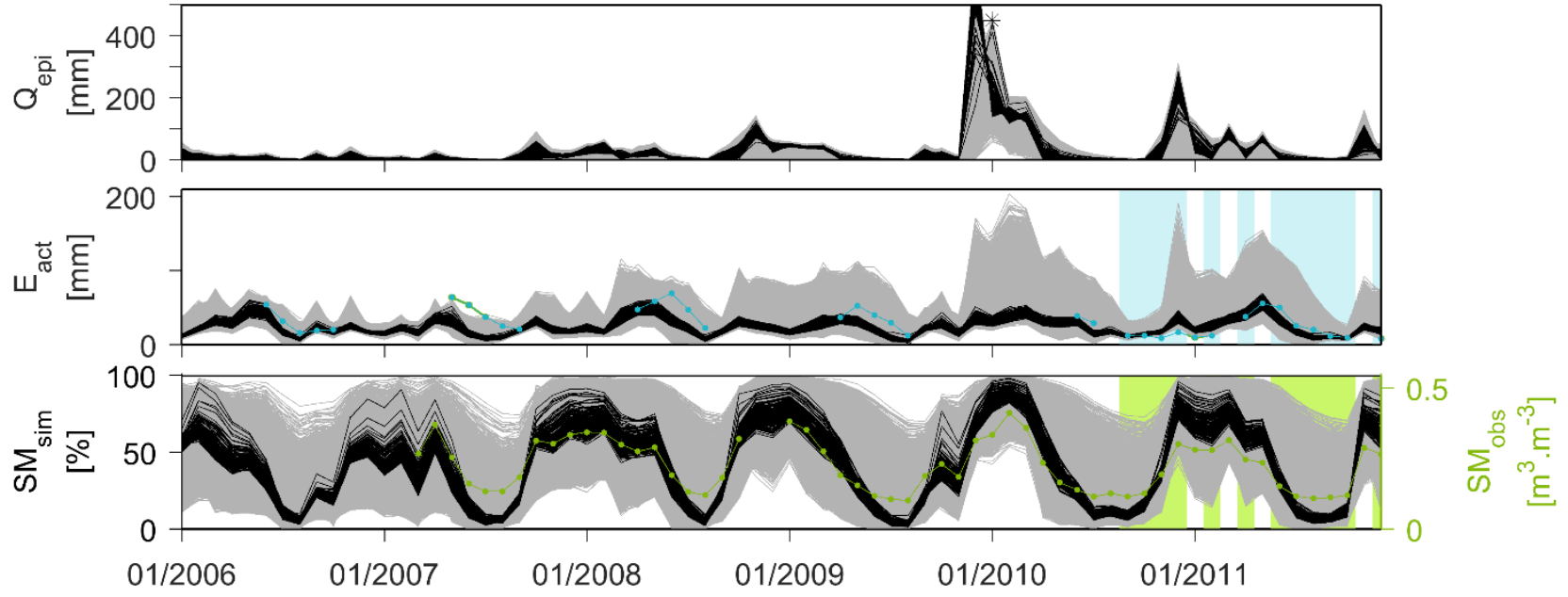

b. hourly
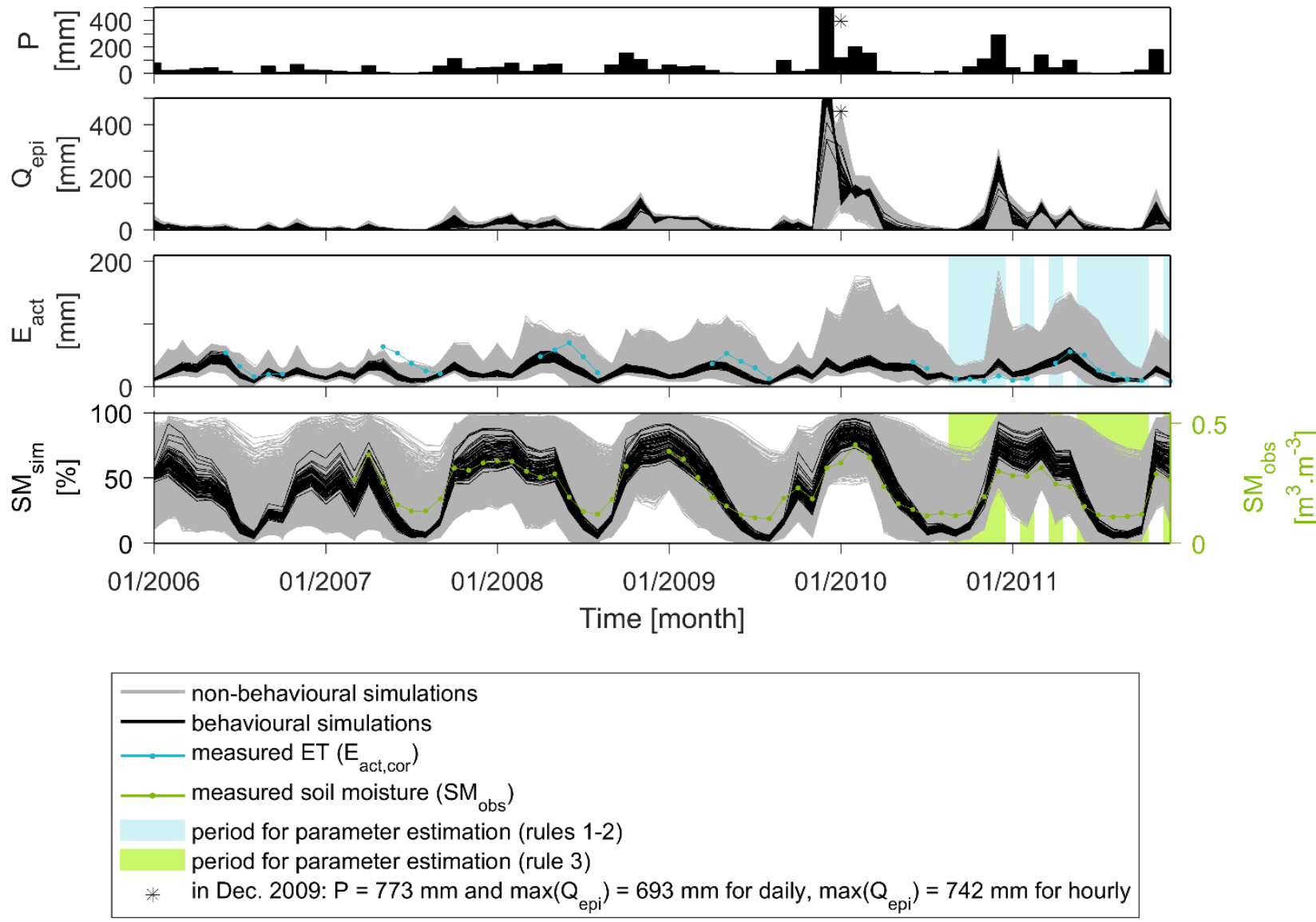

Figure S16. Monthly time series of precipitation input $(P)$, simulated recharge $\left(Q_{e p i}\right)$, simulated actual ET ( $E_{a c t}$, which is the sum of evaporation from canopy interception, transpiration and soil evaporation), simulated soil moisture within the root zone $\left(S M_{\text {sim }}\right)$, and monthly observations of actual ET and soil moisture at the

5 Spanish site for (a) daily time step, (b) hourly time step. Blue and green shaded areas correspond to the periods in which observation of ET and soil moisture respectively were selected to apply the soft rules of Sect. 4.1 (further details on data processing in Sect. 3.2, S4.1 and S4.2). 


\section{a. daily}

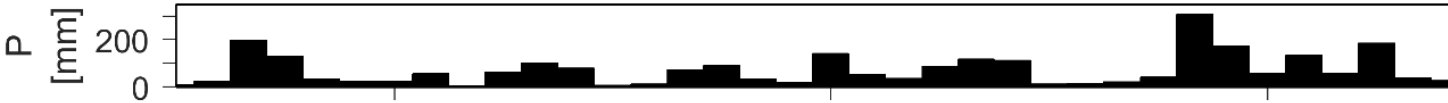
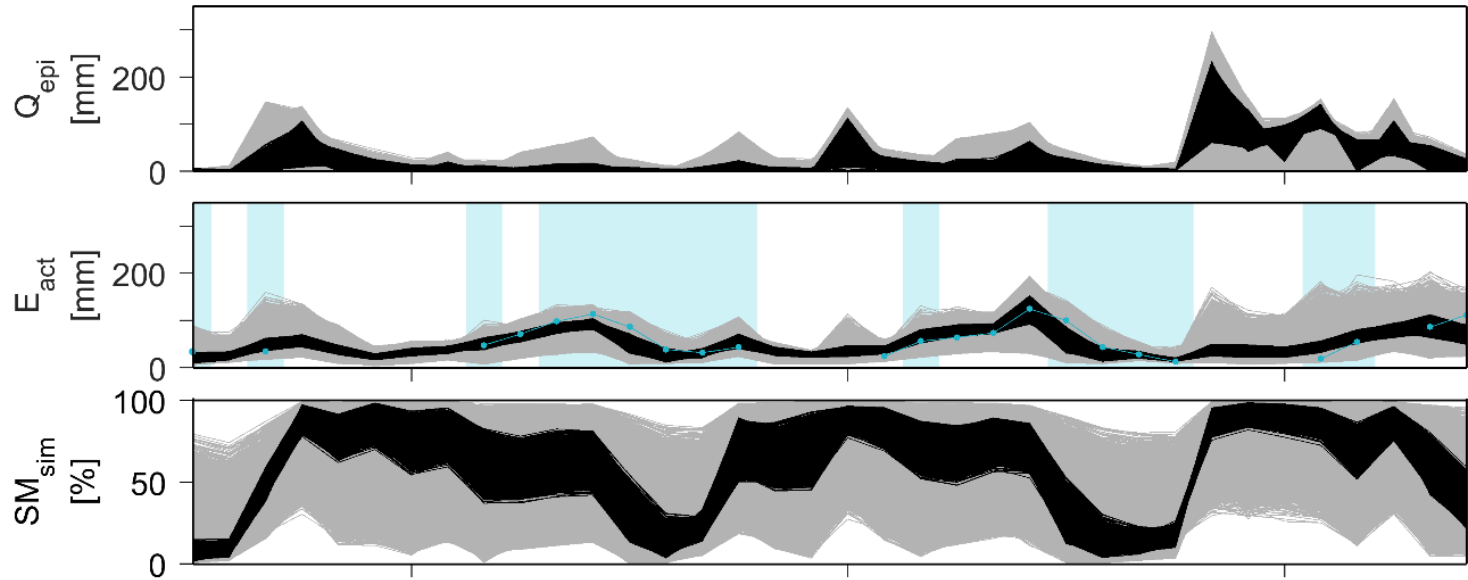

$01 / 2007$

$01 / 2008$

$01 / 2009$

b. hourly
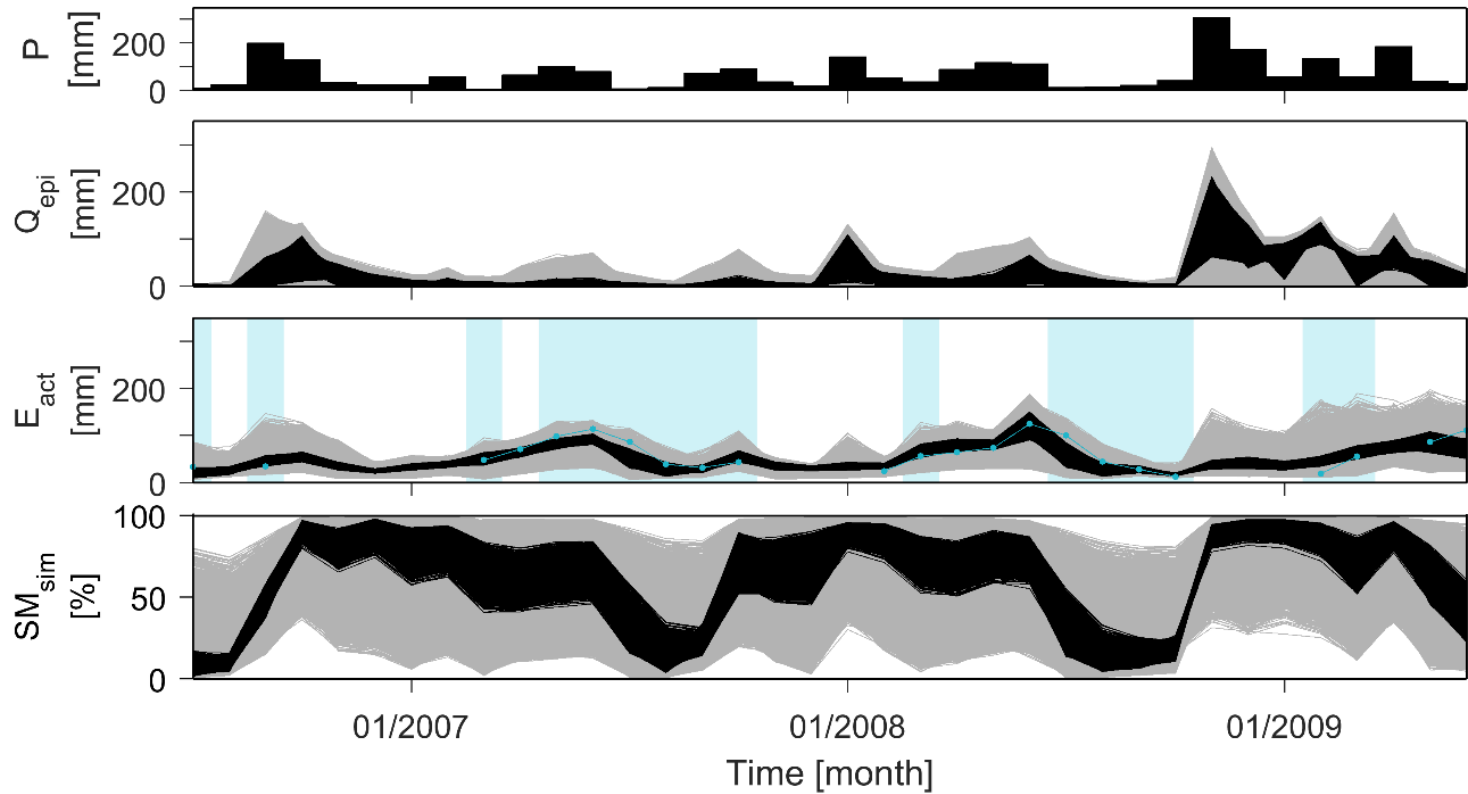

Time [month]

$\begin{aligned}- & \text { non-behavioural simulations } \\ & \text { behavioural simulations } \\ & \text { measured } \mathrm{ET}\left(\mathrm{E}_{\text {act,cor }}\right) \\ & \text { period for parameter estimation (rules 1-2) }\end{aligned}$

Figure S17. Monthly time series of precipitation input $(P)$, simulated recharge $\left(Q_{\text {epi }}\right)$, simulated actual ET ( $E_{a c t}$, which is the sum of evaporation from canopy interception, transpiration and soil evaporation), simulated soil moisture within the root zone $\left(S M_{\text {sim }}\right)$, and monthly observations of actual ET and soil moisture at the

5 German site for (a) daily time step, (b) hourly time step. Blue and green shaded areas correspond to the periods in which observation of ET and soil moisture respectively were selected to apply the soft rules of Sect. 4.1 (further details on data processing in Sect. 3.2, S4.1 and S4.2). 


\section{References}

Allen, R. G., Pereira, L. S., Raes, D. and Smith, M.: Crop evapotranspiration: Guidelines for computing crop requirements, FAO Irrigation and Drainage Paper 56, Food and Agriculture Organization (FAO), Rome, Italy, 1998.

Arnell, N. W.: A simple water balance model for the simulation of streamflow over a large geographic domain, J. Hydrol., 217(3-4), 314-335, doi:10.1016/S0022-1694(99)00023-2, 1999.

Baldocchi, D., Falge, E., Gu, L., Olson, R., Hollinger, D., Running, S., Anthoni, P., Bernhofer, C., Davis, K., Evans, R., Fuentes, J., Goldstein, A., Katul, G., Law, B., Lee, X., Malhi, Y., Meyers, T., Munger, W., Oechel, W., Paw, K. T., Pilegaard, K., Schmid, H. P., Valentini, R., Verma, S., Vesala, T., Wilson, K. and Wofsy, S.: FLUXNET: A New Tool to Study the Temporal and Spatial Variability of Ecosystem-Scale Carbon Dioxide, Water Vapor, and Energy Flux Densities, Bull. Am. Meteorol. Soc., 82(11), 2415-2434, doi:10.1175/1520-0477(2001)082<2415:FANTTS>2.3.CO;2, 2001.

Baldocchi, D. D. and Ryu, Y.: A Synthesis of Forest Evaporation Fluxes - from Days to Years - as Measured with Eddy Covariance, in Forest Hydrology and Biogeochemistry, Synthesis of Past Research and Future Directions, edited by D. F. Levia, D. Carlyle-Moses, and T. Tanaka, Springer Netherlands, Dordrecht., 101-116, 2011.

Bierkens, M. F. P.: Global hydrology 2015: State, trends, and directions, Water Resour. Res., 51(7), 49234947, doi:10.1002/2015WR017173, 2015.

Bohn, T. J. and Vivoni, E. R.: Process-based characterization of evapotranspiration sources over the North American monsoon region, Water Resour. Res., 52(1), 358-384, doi:10.1002/2015WR017934, 2016.

Bonan, G.: Soil Physics, in Ecological Climatology: Concepts and Applications, Cambridge University Press, Cambridge., 143-154, 2015.

Boone, A., Calvet, J.-C. and Noilhan, J.: Inclusion of a third soil layer in a land surface scheme using the forcerestore method, J. Appl. Meteorol., 38(11), 1611-1630, doi:10.1175/15200450(1999)038<1611:IOATSL>2.0.CO;2, 1999.

Breuer, L., Eckhardt, K. and Frede, H. G.: Plant parameter values for models in temperate climates, Ecol. Modell., 169(2-3), 237-293, doi:10.1016/S0304-3800(03)00274-6, 2003.

Coenders-Gerrits, A. M. J., Van der Ent, R. J., Bogaard, T. A., Wang-Erlandsson, L., Hrachowitz, M. and Savenije, H. H. G.: Uncertainties in transpiration estimates, Nature, 506, E1-E2, doi:10.1038/nature12925, 2014.

Deardorff, J. W.: Efficient prediction of ground surface temperature and moisture, with inclusion of a layer of vegetation, J. Geophys. Res., 83(C4), 1889-1903, doi:10.1029/JC083iC04p01889, 1978.

Dickinson, R. E.: Modeling evapotranspiration for three dimensional global climate models, in Climate Processes and Climate Sensitivity, edited by J. E. Hansen and T. Takahashi, American Geophysical Union, Washington, D. C., 1984.

Döll, P., Kaspar, F. and Lehner, B.: A global hydrological model for deriving water availability indicators: Model tuning and validation, J. Hydrol., 270(1-2), 105-134, doi:10.1016/S0022-1694(02)00283-4, 2003.

Essery, R., Best, M. and Cox, P.: MOSES 2.2 Technical Documentation, Berks, UK. [online] Available from: http://www.metoffice.gov.uk/media/pdf/9/j/HCTN_30.pdf, 2001.

Fang, H., Jiang, C., Li, W., Wei, S., Baret, F., Chen, J. M., Garcia-Haro, J., Liang, S., Liu, R., Myneni, R. B., Pinty, B., Xiao, Z. and Zhu, Z.: Characterization and intercomparison of global moderate resolution leaf area index (LAI) products: Analysis of climatologies and theoretical uncertainties, J. Geophys. Res. Biogeosciences, 118(2), 529-548, doi:10.1002/jgrg.20051, 2013.

Fatichi, S. and Pappas, C.: Constrained variability of modeled T:ET ratio across biomes, Geophys. Res. Lett., 44(13), 6795-6803, doi:10.1002/2017GL074041, 2017.

Federer, C. A., Vörösmarty, C. and Fekete, B.: Sensitivity of Annual Evaporation to Soil and Root Properties in Two Models of Contrasting Complexity, J. Hydrometeorol., 4(6), 1276-1290, doi:10.1175/15257541(2003)004<1276:SOAETS>2.0.CO;2, 2003. 
Foken, T., Leuning, R., Oncley, S. R., Mauder, M. and Aubinet, M.: Corrections and Data Quality Control, in Eddy Covariance: A Practical Guide to Measurement and Data Analysis, edited by Aubinet, M., Vesala, T. and Papale, D., Springer Netherlands, Dordrecht, 85-131, 2012.

Gerten, D., Schaphoff, S., Haberlandt, U., Lucht, W. and Sitch, S.: Terrestrial vegetation and water balance Hydrological evaluation of a dynamic global vegetation model, J. Hydrol., 286(1-4), 249-270, doi:10.1016/j.jhydrol.2003.09.029, 2004.

Gosling, S. N. and Arnell, N. W.: Simulating current global river runoff with a global hydrological model: Model revisions, validation, and sensitivity analysis, Hydrol. Process., 25(7), 1129-1145, doi:10.1002/hyp.7727, 2011.

Granier, A., Bréda, N., Biron, P. and Villette, S.: A lumped water balance model to evaluate duration and intensity of drought constraints in forest stands, Ecol. Modell., 116(2-3), 269-283, doi:10.1016/S03043800(98)00205-1, 1999.

Hartmann, A., Gleeson, T., Rosolem, R., Pianosi, F., Wada, Y. and Wagener, T.: A large-scale simulation model to assess karstic groundwater recharge over Europe and the Mediterranean, Geosci. Model Dev., 8(6), 1729-1746, doi:10.5194/gmd-8-1729-2015, 2015.

Jung, M., Reichstein, M., Margolis, H. A., Cescatti, A., Richardson, A. D., Arain, M. A., Arneth, A., Bernhofer, C., Bonal, D., Chen, J., Gianelle, D., Gobron, N., Kiely, G., Kutsch, W., Lasslop, G., Law, B. E., Lindroth, A., Merbold, L., Montagnani, L., Moors, E. J., Papale, D., Sottocornola, M., Vaccari, F. and Williams, C.: Global patterns of land-atmosphere fluxes of carbon dioxide, latent heat, and sensible heat derived from eddy covariance, satellite, and meteorological observations, J. Geophys. Res. Biogeosciences, 116(G3), G00J07, doi:10.1029/2010JG001566, 2011.

Kergoat, L.: A model for hydrological equilibrium of leaf area index on a global scale, J. Hydrol., 212-213, 268-286, doi:10.1016/S0022-1694(98)00211-X, 1998.

Körner, C.: Leaf diffusive conductances in the major vegetation types of the globe, in Ecophysiology of photosynthesis, edited by E. D. Schulze and M. M. Caldwell, Springer Study Edition, vol 100, Springer, Berlin, Heidelberg., 463-490, 1995.

Kroes, J. G., van Dam, J. C., Groenendijk, P., Hendriks, A. R. F. and Jacobs, C. M. J.: SWAP version 3.2: Theory description and user manual, Report 1649, Alterra, Wageningen, The Netherlands., 2008.

Kumar, R., Samaniego, L. and Attinger, S.: Implications of distributed hydrologic model parameterization on water fluxes at multiple scales and locations, Water Resour. Res., 49(1), 360-379, doi:10.1029/2012WR012195, 2013.

Lhomme, J. P., Boudhina, N. and Masmoudi, M. M.: Technical Note: On the Matt-Shuttleworth approach to estimate crop water requirements, Hydrol. Earth Syst. Sci., 18(11), 4341-4348, doi:10.5194/hess-18-43412014, 2014.

Liang, X., Lettenmaier, D. P., Wood, E. F. and Burges, S. J.: A simple hydrologically based model of land surface water and energy fluxes for general circulation models, J. Geophys. Res., 99(D7), 14415-14428, doi:10.1029/94JD00483, 1994.

Martens, B., Miralles, D. G., Lievens, H., van der Schalie, R., de Jeu, R. A. M., Férnandez-Prieto, D., Beck, H. E., Dorigo, W. A. and Verhoest, N. E. C.: GLEAM v3: satellite-based land evaporation and root-zone soil moisture, Geosci. Model Dev., 10(5), 1903-1925, doi:10.5194/gmd-10-1903-2017, 2017.

Masson, V., Champeaux, J. L., Chauvin, F., Meriguet, C. and Lacaze, R.: A global database of land surface parameters at $1-\mathrm{km}$ resolution in meteorological and climate models, J. Clim., 16(9), 1261-1282, doi:10.1175/1520-0442-16.9.1261, 2003.

McCabe, M. F., Ershadi, A., Jimenez, C., Miralles, D. G., Michel, D. and Wood, E. F.: The GEWEX LandFlux project: Evaluation of model evaporation using tower-based and globally gridded forcing data, Geosci. Model Dev., 9(1), 283-305, doi:10.5194/gmd-9-283-2016, 2016.

Miralles, D. G., Gash, J. H., Holmes, T. R. H., De Jeu, R. A. M. and Dolman, A. J.: Global canopy interception from satellite observations, J. Geophys. Res., 115(D16), D16122, doi:10.1029/2009JD013530, 2010.

Miralles, D. G., Holmes, T. R. H., De Jeu, R. A. M., Gash, J. H., Meesters, A. G. C. A. and Dolman, A. J.: Global land-surface evaporation estimated from satellite-based observations, Hydrol. Earth Syst. Sci., 
15(2), 453-469, doi:10.5194/hess-15-453-2011, 2011.

Miralles, D. G., Jiménez, C., Jung, M., Michel, D., Ershadi, A., McCabe, M. F., Hirschi, M., Martens, B., Dolman, A. J., Fisher, J. B., Mu, Q., Seneviratne, S. I., Wood, E. F. and Fernaìndez-Prieto, D.: The WACMOS-ET project - Part 2: Evaluation of global terrestrial evaporation data sets, Hydrol. Earth Syst. Sci., 20(2), 823-842, doi:doi.org/10.5194/hess-20-823-2016, 2016.

Müller Schmied, H., Eisner, S., Franz, D., Wattenbach, M., Portmann, F. T., Flörke, M. and Döll, P.: Sensitivity of simulated global-scale freshwater fluxes and storages to input data, hydrological model structure, human water use and calibration, Hydrol. Earth Syst. Sci., 18(9), 3511-3538, doi:10.5194/hess-18-3511-2014, 2014.

New, M., Lister, D., Hulme, M. and Makin, I.: A high-resolution data set of surface climate over global land areas, Clim. Res., 21(1), 1-25, doi:10.3354/cr021001, 2002.

Noilhan, J. and Planton, S.: A Simple Parameterization of Land Surface Processes for Meteorological Models, Mon. Weather Rev., 117(3), 536-549, doi:10.1175/1520-0493(1989)117<0536:ASPOLS>2.0.CO;2, 1989.

Oleson, K. W., Lawrence, D. M., Bonan, G. B., Flanner, M. G., Kluzek, E., Lawrence, P. J., Levis, S., Swenson, S. C., Peter, Thornton, E., Dai, A., Decker, M., Dickinson, R., Feddema, J., Heald, C. L., Hoffman, F., Lamarque, J.-F., Mahowald, N., Niu, G.-Y., Qian, T., Randerson, J., Running, S., Sakaguchi, K., Slater, A., Stöckli, R., Wang, A., Yang, Z.-L., Zeng, X. and Zeng, X.: Technical Description of version 4.0 of the Community Land Model (CLM), NCAR/TN-478+STR, NCAR technical note, National Center for Atmospheric Research (NCAR), Boulder, Colorado., 2010.

Penman, H. L.: The dependance of transpiration on weather and soil conditions, J. Soil Sci., 1(1), 74-89, doi:10.1111/j.1365-2389.1950.tb00720.x, 1950.

Rakovec, O., Kumar, R., Mai, J., Cuntz, M., Thober, S., Zink, M., Attinger, S., Schäfer, D., Schrön, M. and Samaniego, L.: Multiscale and Multivariate Evaluation of Water Fluxes and States over European River Basins, J. Hydrometeorol., 17(1), 287-307, doi:10.1175/JHM-D-15-0054.1, 2016.

Rodell, M., Houser, P. R., Jambor, U., Gottschalck, J., Mitchell, K., Meng, C.-J., Arsenault, K., Cosgrove, B., Radakovich, J., Bosilovich, M., Entin, J. . K., Walker, J. P., Lohmann, D. and Toll, D.: The Global Land Data Assimilation System, Bull. Am. Meteorol. Soc., 85(3), 381-394, doi:10.1175/BAMS-85-3-381, 2004.

Ruiz, L., Varma, M. R. R., Kumar, M. M. S., Sekhar, M., Maréchal, J.-C., Descloitres, M., Riotte, J., Kumar, S., Kumar, C. and Braun, J.-J.: Water balance modelling in a tropical watershed under deciduous forest (Mule Hole, India): Regolith matric storage buffers the groundwater recharge process, J. Hydrol., 380(34), 460-472, doi:10.1016/j.jhydrol.2009.11.020, 2010.

Salter, P. J. and Williams, J. B.: The influence of texture on the moisture characteristics of soils- II. Available water capacity and moisture release characteristics, J. Soil Sci., 16(2), 310-317, doi:10.1111/j.13652389.1965.tb01442.x, 1965.

Samaniego, L., Kumar, R. and Attinger, S.: Multiscale parameter regionalization of a grid-based hydrologic model at the mesoscale, Water Resour. Res., 46(5), W05523, doi:10.1029/2008WR007327, 2010.

Schenk, H. J. and Jackson, R. B.: The Global Biogeography of Roots, Ecol. Monogr., 72(3), 311-328, doi:10.1890/0012-9615(2002)072[0311:TGBOR]2.0.CO;2, 2002.

Shuttleworth, W. J. and Wallace, J. S.: Evaporation From Spare Crops - An Energy Combination Theory, Q. J. R. Meteorol. Soc., 111(469), 839-855, doi:10.1002/qj.49711146910, 1985.

Šimůnek, J., Šejna, M., Saito, H., Sakai, M. and van Genuchten, M. T.: The HYDRUS-1D software package for simulating the one-dimensional movement of water, heat, and multiple solutes in variably-saturated media, Version 4.08, University of California Riverside, Riverside, USA., 2009.

Sitch, S., Smith, B., Prentice, I. C., Arneth, a., Bondeau, a., Cramer, W., Kaplan, J. O., Levis, S., Lucht, W., Sykes, M. T., Thonicke, K. and Venevsky, S.: Evaluation of ecosystem dynamics, plant geography and terrestrial carbon cycling in the LPJ dynamic global vegetation model , Glob. Chang. Biol., 9(2), 161-185, doi:10.1046/j.1365-2486.2003.00569.x, 2003.

Smith, K. A.: Investigating Uncertainty in Global Hydrology Modelling, Ph.D.thesis, University of Nottingham, Nottingham, UK., 2016.

Sperna Weiland, F. C., Vrugt, J. A., Van Beek, R. L. P. H., Weerts, A. H. and Bierkens, M. F. P.: Significant 
uncertainty in global scale hydrological modeling from precipitation data errors, J. Hydrol., 529(3), 10951115, doi:10.1016/j.jhydrol.2015.08.061, 2015.

Sutanto, S. J., Van Den Hurk, B., Dirmeyer, P. A., Seneviratne, S. I., Röckmann, T., Trenberth, K. E., Blyth, E. M., Wenninger, J. and Hoffmann, G.: HESS Opinions "a perspective on isotope versus non-isotope approaches to determine the contribution of transpiration to total evaporation," Hydrol. Earth Syst. Sci., 18(8), 2815-2827, doi:10.5194/hess-18-2815-2014, 2014.

Sutanudjaja, E. H., Van Beek, L. P. H., De Jong, S. M., Van Geer, F. C. and Bierkens, M. F. P.: Large-scale groundwater modeling using global datasets: A test case for the Rhine-Meuse basin, Hydrol. Earth Syst. Sci., 15(9), 2913-2935, doi:10.5194/hess-15-2913-2011, 2011.

Thornthwaite, C. W.: An Approach toward a Rational Classification of Climate, Geogr. Rev., 38(1), 55-94, doi:10.2307/210739, 1948.

Twine, T. E., Kustas, W. P., Norman, J. M., Cook, D. R., Houser, P. R., Meyers, T. P., Prueger, J. H., Starks, P. J. and Wesley, M. L.: Correcting eddy covariance flux underestimates over grassland, Agric. For. Meteorol., 103(3), 279-300, doi:10.1016/S0168-1923(00)00123-4, 2000.

Van Beek, L. P. H. and Bierkens, M. F. P.: The Global Hydrological Model PCR-GLOBWB: Conceptualization, Parameterization and Verification, Report Department of Physical Geography, Utrecht University, Netherlands. [online] Available from: http://vanbeek.geo.uu.nl/suppinfo/vanbeekbierkens2009.pdf, 2008.

Van Beek, R.: Forcing PCR-GLOBWB with CRU data, Utrecht University, the Netherlands. [online] Available from: http://vanbeek.geo.uu.nl/suppinfo/vanbeek2008.pdf, 2008.

Van de Griend, A. A. and Owe, M.: Bare soil surface resistance to evaporation by vapor diffusion under semiarid conditions, Water Resour. Res., 30(2), 181-188, doi:10.1029/93WR02747, 1994.

Van Dijk, A. I. J. M. and Bruijnzeel, L. A.: Modelling rainfall interception by vegetation of variable density using an adapted analytical model. Part 1. Model description, J. Hydrol., 247(3-4), 230-238, doi:10.1016/S0022-1694(01)00392-4, 2001.

Van Dijk, A. I. J. M., Gash, J. H., Van Gorsel, E., Blanken, P. D., Cescatti, A., Emmel, C., Gielen, B., Harman, I. N., Kiely, G., Merbold, L., Montagnani, L., Moors, E., Sottocornola, M., Varlagin, A., Williams, C. A. and Wohlfahrt, G.: Rainfall interception and the coupled surface water and energy balance, Agric. For. Meteorol., 214-215, 402-415, doi:10.1016/j.agrformet.2015.09.006, 2015.

Vörösmarty, C. J., Moore, B., Grace, A. L., Gildea, M. P., Melillo, J. M., Peterson, B. J., Rastetter, E. B. and Steudler, P. A.: Continental scale models of water balance and fluvial transport: An application to South America, Global Biogeochem. Cycles, 3(3), 241-265, doi:10.1029/GB003i003p00241, 1989.

Vörösmarty, C. J., Willmott, C. J., Choudhury, B. J., Schloss, A. L., Stearns, T. K., Robeson, S. M. and Dorman, T. J.: Analyzing the discharge regime of a large tropical river through remote sensing, groundbased climatic data , and modeling, , 32(10), 3137-3150, doi:10.1029/96WR01333, 1996.

Vörösmarty, C. J., Federer, C. A. and Schloss, A. L.: Potential evapotranspiration functions compared on US watersheds: implications for global-scale water balance and terrestrial ecosystem modeling, J. Hydrol., 207(3-4), 147-169, doi:10.1016/S0022-1694(98)00109-7, 1998.

Wagener, T., McIntyre, N., Lees, M. J., Wheater, H. S. and Gupta, H. V.: Towards reduced uncertainty in conceptual rainfall-runoff modelling: Dynamic identifiability analysis, Hydrol. Process., 17(2), 455-476, doi:10.1002/hyp.1135, 2003.

Wang-Erlandsson, L., Bastiaanssen, W. G. M., Gao, H., Jägermeyr, J., Senay, G. B., Van Dijk, A. I. J. M., Guerschman, J. P., Keys, P. W., Gordon, L. J. and Savenije, H. H. G.: Global root zone storage capacity from satellite-based evaporation, Hydrol. Earth Syst. Sci., 20(4), 1459-1481, doi:10.5194/hess-20-14592016, 2016.

Weedon, G. P., Gomes, S., Viterbo, P., Österle, H., Adam, J. C., Bellouin, N., Boucher, O. and Best, M.: The Watch Forcing Data 1958-2001: a Meteorological Forcing Dataset for Land Surface-and HydrologicalModels. Technical Report 22., 2010.

Wigmosta, M. S., Vail, L. W. and Lettenmaier, D. P.: A distributed hydrology-vegetation model for complex terrain, Water Resour. Res., 30(6), 1665-1679, doi:10.1029/94WR00436, 1994. 
Williams, P. W. and Ford, D. C.: Global distribution of carbonate rocks, Zeitschrift für Geomorphol. Suppl., 147, 1-2, 2006.

Xue, J. and Su, B.: Significant remote sensing vegetation indices: A review of developments and applications, J. Sensors, 2017, Article ID 1353691, doi:10.1155/2017/1353691, 2017.

5 Yang, Z.-L., Cai, X., Zhang, G., Tavakoly, A. A., Jin, Q., Meyer, L. H. and Guan, X.: The Community Noah Land Surface Model with Multi-Parameterization Options (Noah-MP) - Technical description, University of Texas at Austin, Austin, USA. [online] Available from: http://www.jsg.utexas.edu/noah-mp/files/noahmp_technote_v0.2.pdf, 2011. 